\title{
HACIA UNA FILOSOFÍA DE LA ASTROBIOLOGÍA
}

\author{
ROBERTO ARETXAGA-BURGOS \\ Universidad de Deusto, Bilbao
}

\begin{abstract}
RESUMEN: Tomando como fundamento la historia y literatura astrobiológicas, exploramos la posibilidad y necesidad de una Filosofía de la Astrobiología, proponemos una definición de Filosofía de la Astrobiología y su consideración como subdisciplina de la Filosofía de la Ciencia, distinta de la Filosofía de la Biología. Incluimos una bibliografía para una Filosofía de la Astrobiología.

PALABRAS CLAVE: astrobiología, exobiología, biología, filosofía, filosofía de la astrobiología, filosofía de la biología, ética, teología, ciencia, tecnología, sociedad, sentido, comunicación, realidad, definición, disciplina, subdisciplina, vida, inteligencia, extraterrestre, universo, evolución, convergencia, azar, necesidad, imperativo cósmico.
\end{abstract}

\section{Towards a Philosophy of Astrobiology}

ABSTRACT: Based on the history of astrobiology and astrobiological literature, we explore the possibility and necessity of a Philosophy of Astrobiology, and propose a tentative definition of Philosophy of Astrobiology for being considered as a possible sub-discipline of Philosophy of Science, different from Philosophy of Biology. We include a Bibliography for a Philosophy of Astrobiology.

KEY WORDS: astrobiology, exobiology, biology, philosophy, philosophy of astrobiology, philosophy of biology, ethics, theology, science, technology, society, sense, communication, reality, definition, discipline, sub-discipline, life, intelligence, extraterrestrial, universe, evolution, convergence, chance, necessity, cosmic imperative.

\section{Por QuÉ UNA FILOSOFÍA DE LA ASTROBIOLOGÍA}

Aunque presente en los orígenes mismos del pensamiento occidental, la cuestión de la pluralidad de mundos y la posible existencia de vida en ellos ha ocupado, sin embargo, un lugar marginal en el pensamiento filosófico y se halla carente de sistematización.

Durante la época clásica la especulación sobre la posible existencia de mundos habitados estuvo unida a las consideraciones acerca del ser de la naturaleza, es decir, físicas y cosmológicas, tal vg. Epicuro y Lucrecio. En la Edad Media, en cambio, la cuestión pasó a convertirse en un corolario de la reflexión sobre la omnipotencia divina — caso de Etienne Tempier, obispo de París. Por otra parte, interés por la vida en aquella época giraba en torno a la salvación del alma, de modo que la cuestión de la vida en otros mundos quedó velada por la de la vida en el otro mundo.

Fue en el Renacimiento cuando el tema de la pluralidad de mundos y la posible existencia de vida en ellos encontró un contexto más adecuado para su 
desarrollo. El nuevo espíritu científico de la época promovió el avance teórico y técnico de la astronomía, que llevó al descubrimiento de nuevos mundos (Galileo), confirmándose a un nuevo nivel el axioma hermético: lo que es arriba es abajo y lo que es abajo es arriba —en este caso, la similitud entre la estructura del mundo conocido sublunar y los nuevos mundos supralunares descubiertos. Giordano Bruno será la figura paradigmática del nuevo enfoque.

En el siglo xx, especialmente durante la segunda mitad, se sucedieron rápidos y grandes avances en las ciencias de la vida - particularmente en biología molecular y genética-, en informática, astrofísica, astronomía y astronáutica. Esta acumulación de circunstancias posibilitó que la cuestión de la vida en otros mundos alcanzara una fase de afianzamiento no solo distinta, sino diversa de la anterior. Así, la química prebiótica — nacida con el experimento de Urey-Miller (1953), en el que obtuvieron algunos compuestos básicos para la vida - fue incorporada de inmediato al incipiente programa espacial estadounidense, surgiendo una nueva área de interés científico: los estudios de la vida más allá de la Tierra (Exobiología). El primer proyecto astrobiológico — exobiológico- de la NASA data de 1959, y en 1960 Joshua Lederberg - Nobel de Fisiología y Medicina en 1958- publicó el escrito seminal «Exobiology: Approachess to Life Beyond the Earth»'.

Advertida la necesidad de revisar sus fundamentos y estrategias, en los años 90 del siglo pasado tiene lugar la refundación de la Astrobiología, creándose en 1995 el AMES Research Center (NASA), y en 1998 el NASA Astrobiology Institute (NAI), organismo que lidera actualmente los estudios astrobiológicos en el mundo. España se sumó pronto a esta iniciativa, creando en 1999, tras un intercambio de contactos a nivel gubernamental, el Centro de Astrobiología (CAB), un Organismo Público de Investigación (OPI) que contó desde el comienzo — no sin la oposición de algunos científicos- con la participación del Consejo Superior de Investigaciones Científicas (CSIC). Ese mismo año - formalmente en 2000-, el CAB obtuvo el estatus de asociado al Instituto de Astrobiología de la NASA, siendo el primer centro de excelencia no estadounidense en vincularse al $\mathrm{NAI}^{2}$.

El establecimiento del programa exobiológico de la NASA y el citado escrito de Lederberg generaron de forma inmediata y natural dos consecuencias de carácter filosófico: la primera, un debate epistemológico — suscitado principalmente por sus detractores, en parte por recelos profesionales-, acerca de la identidad de la nueva disciplina emergente y su estatuto científico; la segunda,

1 En 1960 Joshua Lederberg intervino en el Primer Simposio de Ciencia Espacial del COSPAR, celebrado en Niza (Francia). En dicho Simposio, Lederberg reivindicó un lugar entre las ciencias para una nueva disciplina, a la que denominó exobiología. El contenido de su intervención apareció publicado el 12 de agosto de ese mismo año en la revista Science, constituyendo el mencionado escrito seminal.

2 Posteriormente se vincularon en calidad de colaboradores organismos como el británico United Kingdom Astrobiology Forum and Network, el Australian Centre for Astrobiology, el Groupement de Recherche en Exobiologie del Centro Nacional de Investigación Científica (CNRS) francés o la European Exo/Astrobiology Network Association (EANA). 
de carácter ontológico, al incidir en el debate filosófico y científico sobre qué sea la vida.

En definitiva, la cuestión de la pluralidad de mundos habitados, sobre la que la tradición filosófica había venido especulando desde sus comienzos, cobra interés científico a mediados del siglo xx, y lo hace, además, provocando una encendida discusión epistemológica entre científicos acerca de la actividad destinada a ocuparse de su estudio - la exobiología-, a la vez que suscitando cuestiones ontológicas dadas sus implicaciones para la comprensión científica de la vida y su definición.

Puesto que nos hallamos en la época fundacional de la Astrobiología, también lo estamos en la de la Filosofía de la Astrobiología. Mientras la Astrobiología/Exobiología se halle en fase emergente, también lo estará la Filosofía de la Astrobiología, es decir, la meta-reflexión sobre dicha actividad científica y su objeto. En la medida en que la Astrobiología logré consolidarse y actuar, esta disciplina suscitará y afrontará de modo creciente e inevitable cuestiones filosóficas, evidenciando progresivamente la necesidad de que la Filosofía se interese por su estructura, actividad, problemas, implicaciones y consecuencias. Buena prueba de lo afirmado es la frecuencia con que los astrobiólogos reclaman desde hace tiempo, y de forma creciente, la presencia de la filosofía y de los filósofos en su campo. Es fácil encontrar en la literatura astrobiológica, y hasta en la información universitaria, ejemplos de ello³.

3 Véanse a título de ejemplo las siguientes citas: "Como tal, la exobiología descansa sobre la intersección de áreas tan tradicionales como la geología, la astronomía, la ciencia planetaria, la química y la biología. Se trata, por tanto, de una rama interdisciplinar que toca todos los aspectos de la ciencia. Y, dadas las implicaciones de los resultados arrojados por la búsqueda de vida extraterrestre, también guarda relación con cuestiones que por lo general conciernen a la filosofía, la teología y otros campos del saber que no suelen formar parte de las ciencias naturales» (JАKоSKY, B., La búsqueda de vida en otros planetas. Madrid: Cambridge University Press, 1999, p. 14); "La posibilidad de que haya vida en algún otro lugar del Sistema Solar o del universo constituye una de las cuestiones más profundas que podemos plantearnos. Sea cual sea la respuesta a tal pregunta (la presencia o la ausencia de vida en otra parte), afectará de manera espectacular a nuestra concepción del mundo y del lugar que ocupamos en él. El hallazgo de un solo ejemplo de vida elemental en otro mundo bastará para comunicarnos que el surgimiento de la vida no es exclusivo de la Tierra y no podremos por menos que preguntarnos si existe vida inteligente más avanzada o más sofisticada que la nuestra. En tal caso nos plantearemos si los caminos que hemos elegido como sociedad son o no los más adecuados. De hecho, el descubrimiento de que no estamos solos en nuestra existencia nos depararía toda una experiencia de humildad. Por otra parte, si no encontráramos ninguna prueba de que haya otras formas de vida, contemplaríamos la Tierra y todos sus habitantes como un fenómeno excepcional, ubicado en un plano exclusivo en el seno del vasto universo» (op. cit., p. 11)

Por otra parte, los Departamentos de Filosofía de algunas universidades norteamericanas informan ya a sus estudiantes —o futuros estudiantes — del tirón de la Astrobiología y las oportunidades profesionales que ofrece el tándem filosofía-astrobiología, ante la necesidad de la NASA de contar con expertos en epistemología y ética, principalmente, dadas las consecuencias filosóficas que plantean cuestiones como el futuro y expansión de la vida en el Universo. Tal es el caso de la nota firmada por Mark Lupisella (NASA Goddard Space Flight 
En la actualidad, la idea de que algún día — quizá no muy lejano- pueda hallarse vida extraterrestre, incluso inteligente, comienza a gozar de respetabilidad científica, siendo este cambio de actitud y mentalidad mérito de la Astrobiología. No será ejercicio baldío, por tanto, prepararse para semejante eventualidad, crucial por múltiples razones para la cultura humana, y que validaría definitivamente la Astrobiología como ciencia, comenzando entonces su verdadera labor y vocación.

En ese hipotético momento resultaría patente la necesidad y utilidad de la reflexión filosófica sobre la cuestión, pero también el azoramiento en el caso de no haberla previsto con la suficiente antelación o, por contra, el alivio de quien ya dispone de una herramienta eficaz para enfrentar tal contingencia, y hacerlo con serenidad. Por ello, no encuentro mejor explicación de lo que a día de hoy sería el interés de una Filosofía de la Astrobiología, ni mejor expresada, que lo dicho por Ortega y Gasset en cierta ocasión a propósito de otro asunto: «Siempre he considerado que la misión del escritor es prever con holgada anticipación lo que va a ser problema, años más tarde, para sus lectores y proporcionarles a tiempo, es decir, antes de que el debate surja, ideas claras sobre la cuestión, de modo que entren en el fragor de la contienda con el ánimo sereno de quien, en principio, ya la tiene resuelta» ${ }^{4}$.

Todo lo anteriormente expuesto pone de manifiesto y justifica, a nuestro juicio, la necesidad de comenzar ya la construcción de una Filosofía de la Astrobiología, labor a la que este escrito pretende contribuir.

\section{Principal dificultad Para la elaboración de una Filosofía DE LA ASTROBIOLOGÍA EN LA ACTUALIDAD}

La principal dificultad u objeción para la construcción y consolidación de una Filosofía de la Astrobiología en la actualidad —más aún si pretende ser sistemática- radica, a nuestro juicio, en la definición o delimitación del sujeto sobre el que debe desarrollar su actividad: la Astrobiología. ¿Qué es la Astrobiología? ¿Es ciencia la Astrobiología? ¿Posee la Astrobiología realmente objeto de estudio? Hemos mantenido en otros lugares que la Astrobiología en su fase

Center), que a día de hoy (07/07/2014), figura en la web del Dpto. de Filosofía de la Universidad de Mariland, USA (cf. www.philosophy.umd.edu/undergraduate/alumni).

Finalmente, nosotros mismos pudimos comprobar personalmente este particular durante una conversación informal mantenida con Frank Drake y el físico y matemático Julián Chela-Flores, con ocasión de la celebración de la VII Conferencia Internacional sobre el Origen de la Vida (Trieste, 2003). Durante la charla, y sabiendo que yo era filósofo y el profesor Chela-Flores se interesaba por los aspectos filosóficos de la Astrobiología, el insigne astrónomo estadounidense aprovechó para incidir en los aspectos filosóficos de la astrobiología, así como en la necesidad de aumentar la presencia y participación de mis colegas en este campo.

4 Ortega y Gasset, J., Meditación de la técnica. Madrid: Revista de Occidente, 1957, 3ª , p. 5 . 
actual no es todavía una ciencia bien establecida, sino una exploración científi$\mathrm{ca}^{5}$. En consecuencia, una Filosofía de la Astrobiología será una filosofía sobre una actividad exploratoria ¿Cómo pretender que tal reflexión se constituya en subdisciplina académicamente reconocida?

En relación con este problema, resulta particularmente significativa desde nuestra perspectiva una afirmación vertida por Grinspoon en su obra Lonely Planets: "The odd status of astrobiology in the suite of sciences can, I think, be understood by realizing that it is not yet science, exactly, but still natural philosophy». La consideración del científico estadounidense de la Astrobiología como filosofía natural, antes que como ciencia, pone de manifiesto, por una parte, la dificultad arriba apuntada y, por otra, la necesidad de lo que aquí proponemos: una reflexión filosófica sistemática sobre la Astrobiología, es decir, la construcción de una Filosofía de la Astrobiología susceptible de reconocimiento académico.

En la actualidad, la Filosofía de la Astrobiología se encuentra en fase de escorzo, de intentos y tanteos, de reflexiones faltas de sistematización sobre temas y aspectos parciales. Con todo, es posible encontrar algunos esfuerzos que apuntan en el sentido de nuestra propuesta, tanto en el ámbito de habla inglesa como en el hispanoparlante ${ }^{6}$.

\section{Hacia una FILOSOFía de la ASTRobiología}

Ante la dificultad apuntada — delimitación del objeto de una Filosofía de la Astrobiología- estableceremos la siguiente estrategia:

1) Elaboración de una definición razonada, pragmática u operativa, para la expresión Filosofía de la Astrobiología;

2) Identificación de los principales campos, disciplinas y subdisciplinas filosóficos implicados con la Astrobiología, tomando para ello como referencia los diferentes aspectos, temas y cuestiones de interés filosófico abordados en la literatura astrobiológica;

5 Cf. «La Astrobiología como exploración científica», Hacia el Espacio. Revista de la Agencia Espacial Mexicana (AEM), n 15 (Especial monográfico sobre astrobiología), junio de 2014; "Cultural Implications of the Search and Eventual Discovery of a Second Genesis», en Seсквасн, J. (ed.): Genesis - In The Beginning. Precursors of Life, Chemical Models and Early Biological Evolution, Dordrecht (The Netherlands): Springer, Series Cellular Origin, Life in Extreme Habitats and Astrobiology (COLE), 2012, Vol. 22, pp. 873-890; «Astrobiología: entre la ciencia y la exploración», Letras de Deusto (Universidad de Deusto, Bilbao), ${ }^{\circ} 118$, Vol. 38 (2008), pp. 13-27.

6 Uno de los más reciente en el ámbito de habla inglesa es Dunér, D. et al., The History and Philosophy of Astrobiology. Newcastle upon Tyne: Cambridge Scholars Publishing, 2013. En el de habla hispana, y cuando menos único en España, las cuatro entregas de la Serie Astrobiología y Filosofía, promovida y dirigida por ARETXAGA BuRgos, R., aparecida periódicamente en la revista universitaria Letras de Deusto (UD, Bilbao) entre 2003 y 2012. 
3) Validación de la definición del punto i) a la luz de los resultados obtenidos en el punto ii), ofreciendo todo ello como contribución a la elaboración de una Filosofía de la Astrobiología.

Completaremos la tarea ofreciendo una selección de obras y escritos de literatura astrobiológica en los que se tratan en detalle las cuestiones, problemas y aspectos esbozados en el punto ii).

\subsection{Elaboración de una definición operacional de Filosofía de la Astrobiología}

Dados: a) el carácter emergente de la Astrobiología; b) el creciente y acelerado desarrollo experimentado por ésta, particularmente en la dos últimas décadas; y c) el empleo cada vez más frecuente en la literatura astrobiológica de la expresión Filosofía de la Astrobiología —en adelante FAB-, urge definir y fijar su significado, lo que constituye ya en sí misma una tarea netamente filosófica.

Comenzaremos esta labor atendiendo, primeramente, al objeto sobre el que se pretende hacer filosofía - la Astrobiología-, para luego hacer lo propio con el término Filosofía. Partiremos para ello del uso de los términos con el fin de evitar que nos sobrevengan en este momento problemas añadidos que dificulten innecesariamente nuestra labor.

\subsubsection{Definición de Astrobiología}

La edición más reciente, a fecha de este escrito, del Diccionario de la RAE (DRAE, 22 2001) ${ }^{7}$ no recoge el término Astrobiología. Deberemos acudir, por tanto, al ámbito especializado —el astrobiológico- para encontrarle un significado.

En la presentación de la Astrobiología que hace la página web del Centro de Astrobiología ( $\mathrm{CAB}$ ) —institución de referencia en España para esta disciplina-, se dice: «No hay una definición consensuada de astrobiología, aunque su campo de interés es perfectamente reconocible» ${ }^{8}$. Quien esto afirma es Juan Pérez Mercader, ex-Director de la Institución. Pero, si la Astrobiología constituye

7 En enero de 2004 comienzan las actualizaciones en línea al DRAE. La primera fue publicada en Internet el 15 de enero de 2004, con 2576 cambios correspondientes al período 17/10/2001-26/03/2003. La segunda se incorpora el 21/04/2005, con 9029 modificaciones correspondientes al período 26/03/2003-24/06/2004. El 30 de octubre de 2007 sigue una nueva actualización, con 4618 cambios datados entre el 24/06/2004 y el 13/12/2006. El 30 de julio de 2010, con 2996 novedades aprobadas entre 13/12/2006 y 28/06/2007, hay una nueva entrega. Y finalmente, la quinta y última es del día 22 de junio de 2012, con una selección de 1697 cambios correspondientes al período 28/06/2007-30/06/2011. Ninguna de estas actualizaciones recoge la entrada «Astrobiología». Última consulta realizada para el presente escrito: 03/06/2014.

8 Cf. http://www.cab.inta.es/es/astrobiologia Web consultada por última vez el día 03/06/2014. 
un campo perfectamente reconocible $e^{9}$ ¿ ¿qué impide, entonces, el consenso en su definición? Plantear esta cuestión es también una labor filosófica, como tendremos ocasión de mostrar más adelante.

Con todo, se hace preciso delimitar el significado de los términos. Álvaro Giménez Cañete, por su parte, en la introducción a una reciente obra divulgativa de la Astrobiología publicada por el CSIC, la define como «el área de la ciencia que estudia el origen de la vida, su supervivencia y distribución en el contexto del Universo» ${ }^{10}$.

El uso del término Astrobiología parece imponerse a partir de 1995, cuando el Administrador Adjunto de la NASA, W. T. Huntress Jr. lo emplea para referirse al nuevo programa para el estudio de la vida en el Universo elaborado por la agencia espacial americana. Sin embargo, en un sentido moderno, el término fue usado ya con anterioridad por el astrónomo soviético Gabriel Tikhov, en 1953, y aún antes por Lefleur, en $1941^{11}$. La autoría del término Astrobiología parece deberse, sin embargo, a René Berthelot, quien en los años 30 del siglo pasado lo empleó con un significado bien distinto del que posee en la actualidad, para hacer referencia a una suerte de pensamiento astrológico ${ }^{12}$.

En 2002, la NASA definió la Astrobiología como «el estudio del origen, evolución, distribución y futuro de la vida en el universo » ${ }^{13}$; definición que se mantiene en la actualidad ${ }^{14}$, y la más aceptada directa o indirectamente en la literatura astrobiológica. Aunque no exenta de dificultades, como ya hemos mostrado en otros lugares ${ }^{15}$, esta definición debidamente matizada resulta aceptable, siendo la que adoptemos como referencia en este escrito.

9 El carácter reconocible aludido se concreta en lo siguiente: «además de todo lo que tiene que ver con la comprensión del fenómeno de la vida tal y como lo conocemos (su emergencia, condiciones de desarrollo, adaptabilidad — extremofilia—, etc.), también involucra la búsqueda de vida fuera de la Tierra (exobiología) y sus derivaciones, como son la exploración espacial o la planetología» (idem sup.).

10 Giménez Cañete, A., Gómez-Elvira, J. y Martín Mayorga, D. (coords.), Astrobiología. Sobre el origen y evolución de la Vida en el Universo. Madrid: CSIC-Los libros de la Catarata, 2011, p. 15.

11 Tikhov, G., Astrobiology. Moscow (Russia): Molodaya Gvardia Publishing House, 1953; Lafleur, L. J., "Astrobiology», Astronomical Society of the Pacific Leaflet (Jan. 1941), n ${ }^{\circ}$ 143, pp. 333-340.

12 Cf. Lemarchand, G. A., «Una breve historia social de la astrobiología en Iberoamérica», pp. 29-30, en Lemarchand, G. A y Tancredi, G. (eds.), Astrobiología: del Big Bang a las Civilizaciones. Montevideo: UNESCO, 2010, pp. 23-52.

13 "Astrobiology is the study of the origins, evolution, distribution, and future of life in the universe» (NASA Astrobiology Roadmap, Introduction. Final Version, 20/11/2002):

https://astrobiology.nasa.gov/media/medialibrary/2013/09/AB_roadmap_2008.pdf

14 Cf. http://astrobiology.nasa.gov/about-astrobiology/

15 En este momento indicaremos únicamente que, en nuestra opinión, la Astrobiología, si desea justificar su peculiaridad y no resultar prescindible, ha de incluir en su definición el aspecto exobiológico; aspecto que se halla implícito en la aquí adoptada. Paradójicamente, la Exobiología, al contrario que la Astrobiología, sí posee su propio Código UNESCO -2512.01—, en tanto que subdisciplina de las Ciencias del Espacio -2512 - incluida en el campo Ciencias de la Tierra y el Espacio - 25. Pero el diccionario de la RAE tampoco recoge 
Una estrategia complementaria de aproximación al significado del término Astrobiología - menos aristotélica que la anterior, más kantiana- es la de presentarla sirviéndose de una batería de preguntas que delimiten su actividad y campo de acción. Así, vg.: «Algunas de las cuestiones que trata de responder la Astrobiología son las siguientes: ¿Qué es la vida? ¿Cómo surgió la Vida en la Tierra? ¿Cómo evoluciona y se desarrolla? ¿Hay vida en otros lugares del Universo? ¿Cuál es el futuro de la vida en la Tierra y en otros lugares? ${ }^{16}$.

\subsubsection{Definición de Filosofía}

A lo largo de sus xxv siglos de existencia, el término Filosofía ha sido empleado - y continúa siéndolo- con distintos y muy diversos significados en función de épocas, escuelas y autores. A pesar de ello, la Filosofía se encuentra académicamente reconocida y sólidamente asentada en la cultura occidental. Es evidente, por tanto, que la falta de consenso en su definición no le ha impedido, sin embargo, constituirse como un campo definido y reconocible del saber humano, etiquetado actualmente con el código 72 de UNESCO. El hecho tiene sin duda sus implicaciones, y es significativo, para el caso de la Astrobiología.

Por razones prácticas para nuestro propósito, adoptaremos la acepción $\mathrm{n}^{\circ}$ 1 ofrecida por el DRAE en su ultima edición para la entrada Filosofía: «Conjunto de saberes que busca establecer, de manera racional, los principios más generales que organizan y orientan el conocimiento de la realidad, así como el sentido del obrar humano» ${ }^{17}$.

Tomando como punto de partida esta definición, diremos que la Filosofía consiste en una reflexión de segundo nivel —meta-reflexión o meta-saberacerca del conocimiento - de su naturaleza, métodos y resultados- y el sentido de la acción humana.

\subsubsection{Definición de Filosofía de la Astrobiología}

Tomando como referencia las anteriores definiciones de Filosofía y Astrobiología, así como la clasificación de los saberes humanos establecida por UNESCO, proponemos la siguiente definición operacional de Filosofía de la Astrobiología: «subdisciplina filosófica, perteneciente a la disciplina de la Filosofía de la Ciencia, que hace de la Astrobiología su objeto de estudio al reflexionar

la entrada «Exobiología», término acuñado a finales de los 50 por J. Lederberg en el escrito varias veces mencionado, y que fue la denominación inicial de lo que hoy conocemos como Astrobiología. Sobre esta cuestión algo diremos más adelante. Para mayores detalles sobre la discusión acerca del aspecto exobiológico de la Astrobiología y de su carácter clave para su definición, cf. los escritos referenciados en la nota 5.

16 Página web del CAB (presentación de Juan Pérez Mercader), consultada el 03/06/2014. Lo mismo puede observarse en el NASA Astrobioloy Roadmap, o en GimÉnEz CaÑETE et al. (loc. cit.), p. 15.

17 Diccionario de la lengua española (DRAE), 22. ${ }^{\mathrm{a}}$ ed. (2001) 
racionalmente sobre su condición de conocimiento, su práctica, resultados y consecuencias». En concreto, una Filosofía de la Astrobiología se ocuparía de cuestiones tales como los aspectos epistemológicos y metodológicos de la Astrobiología, sus peculiaridades explicativas y problemas conceptuales, así como de la validez y alcance de las implicaciones y consecuencias epistémicas, gnoseológicas, éticas y antropológicas, entre otras, tanto de su práctica como de sus resultados.

No es una definición neutral — vg. la sitúa como subdisciplina filosóficapero el resto del trabajo mostrará si los presupuestos más o menos implícitos que contiene están suficientemente justificados.

\subsection{Principales disciplinas y subdisciplinas filosóficas implicadas con la Astrobiología}

Existen actualmente numerosas instituciones dedicadas al impulso y desarrollo de la Astrobiología, y su número aumenta con rapidez por todo el mundo. Igualmente crece el número de científicos que orientan su labor y conocimientos especializados hacia el área astrobiológica. Paralelamente, aumenta la cantidad de talleres, reuniones científicas, foros, cursos y programas astrobiológicos, así como los recursos destinados al desarrollo de la investigación astrobiológica. Finalmente, es constatable una abundante y creciente producción literaria astrobiológica en el mundo.

A pesar de ello, la Astrobiología carece por ahora de reconocimiento académico, de modo que no existe el grado o licenciatura en Astrobiología. Quien desee ser astrobiólogo deberá cursar primero otros estudios y especialidades para posteriormente aproximarse a la Astrobiología a través de estudios de postgrado. Esta es una de las consecuencias de asistir como testigos al momento fundacional de la Astrobiología. En los últimos años se ha hecho evidente la proliferación de cursos y postgrados o especializaciones de contenido astrobiológico en diversas instituciones universitarias y científicas de nuestro entorno cultural, incluida España ${ }^{18}$. Resulta innegable, por tanto, el auge de la Astrobiología como actividad científica, lo que no deja de contrastar con el hecho de que no haya alcanzado aún su principal y más genuino objetivo, y también el más polémico: el hallazgo de vida extraterrestre (carácter exo-biológico).

18 La Universidad de Washington, por ejemplo, ofrece desde 1999 un certificado de postgrado en astrobiología y una especialización en alguna ciencia particular junto con la mención en Astrobiología. En España comienza a observarse un aumento de ofertas, siendo una de las últimas y más innovadoras la del Ilustre Colegio Oficial de Geólogos de España, que ha organizado recientemente, con la colaboración de la Red Española del Planetología y Astrobiología (REDESPA), el «I Curso online de Planetología y Astrobiología», a impartir entre el 15 de octubre y el 30 de noviembre de 2014, con un total de 100 horas repartidas en 3 módulos con 18 unidades temáticas. En cualquier caso no existe una carrera o grado de Astrobiología/Exobiología. 
En definitiva, si bien existe un innegable y creciente interés en la comunidad científica internacional por la posible existencia de vida en otros lugares del universo distintos de nuestro planeta, y su búsqueda es ya un hecho, su hallazgo, en cambio, es todavía una expectativa. Esta situación es relevante para nuestras consideraciones acerca de las implicaciones y aspectos filosóficos de la Astrobiología, por lo que distinguiremos entre los relativos al estudio de la vida en la Tierra y su búsqueda en otros lugares del Universo, y los de un eventual hallazgo de vida fuera de nuestro planeta.

\subsubsection{Aspectos filosóficos de la búsqueda científica de vida fuera de la Tierra}

En este epígrafe mostraremos cómo la existencia misma de la actividad astrobiológica es ya una fuente de cuestiones filosóficas, con independencia del hecho de que hasta el presente carezcamos de evidencia alguna indiscutible acerca de la existencia de un tipo de vida diferente del albergado por nuestro planeta.

\subsubsection{Los orígenes de la Astrobiología}

La Astrobiología es una actividad surgida, impulsada y apoyada por un entorno social y cultural muy concreto: los Estados Unidos de América y su agencia espacial, NASA, fundada en 1958. La Astrobiología, denominada Exobiología en aquella época, surge a la vez que lo hace la NASA. En julio de 1959, el Administrador General de la agencia espacial norteamericana creó el Bioscience Advisory Committee, organismo que apoyó la creación, en marzo de 1960, de la Office of Life Sciences. En agosto de ese mismo año, la NASA autorizó al Jet Propulsion Laboratory el estudio de una misión no tripulada a Marte en busca de vida en su superficie, la cual se materializó en 1976 con las sondas Viking. El impulso definitivo de la Astrobiología se produjo entre 1995 y 1998, con la creación del AMES Research Center y el NASA Astrobiology Institute (NAI), respectivamente, que lideran mundialmente esta área de estudios

Es claro, por tanto, que la Astrobiología surge en un entorno cultural muy concreto —occidental—, fuertemente institucionalizado — científico-tecnológico-, dentro, además, del contexto histórico de la guerra fría y la consecuente carrera espacial. Todos ellos son aspectos que marcan inevitablemente su naturaleza como disciplina y actividad científica, por lo que algunos autores se han interesado en el análisis de sus presupuestos ideológicos e intereses movilizadores, su relación con los poderes político, económico y militar, o las rivalidades académicas y personales que suscitó - y suscita - la nueva disciplina emergente que, muy marcada en sus orígenes por la biología molecular y la bioquímica, fue vista como una amenaza por otras sólidamente establecidas en el panorama científico de la época ${ }^{19}$.

19 En sus comienzos, la exobiología fue vista con recelo por otras ciencias de la vida bien establecidas, que la consideraban una amenaza para su status. En este sentido, resulta 
Así, pues, el origen mismo de la Astrobiología es ya una cuestión que involucra diversos aspectos concernientes, cuando menos, a la Ética de la Ciencia, la Filosofía de la Ciencia, la Filosofía Social, la Sociología de la Ciencia y la Filosofía de la Técnica.

Y todo ello sin olvidar que, a pesar del énfasis de este epígrafe en el carácter actual de la Astrobiología, los temas y cuestiones filosóficos que ésta plantea se remontan, como indicamos en su momento, hasta los orígenes del pensar occidental mismo, por lo que también incumbe a la Historia de la Filosofía.

\subsubsection{La Astrobiología como disciplina científica}

Como ya quedó indicado, la NASA, en su intento por delimitar el campo de acción de la Astrobiología, definió esta disciplina como el estudio del origen, evolución, distribución y futuro de la vida en el Universo. Pero dado su carácter emergente, la Astrobiología deberá justificar su existencia y validez, al mismo tiempo que posicionarse entre las ciencias o disciplinas científicas ya existentes, especialmente respecto de aquellas que tradicionalmente han hecho de la vida su objeto de estudio e interés. Por ello, en la literatura astrobiológica aparecen discusiones acerca de la necesidad de establecer los límites de la Astrobiología frente a otras disciplinas, aclarar sus vínculos con la Exobiología ${ }^{20}$, descubrir los presupuestos tras cada definición de esta disciplina y decidir acerca de la adecuación o validez de las mismas.

En la definición de la Astrobiología, además de la acción definidora misma —qué sea definir-, aparecen involucrados otros aspectos filosóficos relevantes, tales como la fijación/definición de su objeto de estudio (vida, vida extraterrestre), así como la naturaleza y existencia del mismo, pues pudiera suceder que tal objeto no existiera y, en consecuencia, que tanto la disciplina que de él se ocupa como el término que la designa fueran vacíos. Esta consideración hace que en la definición de Astrobiología se halle implícito el problema del estatuto científico del definiendum.

El problema de la definición de Astrobiología involucra, por tanto, cuando menos, aspectos que interesan a la Lógica, la Ontología, la Epistemología, la Filosofía de la Ciencia y la Filosofía del Lenguaje.

significativo que, en sus orígenes, el programa de la NASA para las ciencias de la vida, tutelado por Lederberg, incluyera solamente biólogos moleculares y bioquímicos. Ello explicaría, al menos en parte, la virulencia de la crítica que el biólogo evolucionista Gaylord Simpson dirigió en su momento contra el programa exobiológico y los exobiólogos, a los que se denominó ex-biólogos.

20 Como ya se indicó - cf. nota 15-, al igual que sucede con el de «Astrobiología», tampoco el vocablo «Exobiología» aparece recogido en el DRAE. Por su parte, la clasificación UNESCO de las ciencias no contempla la Astrobiología, pero sí la Exobiología, que aparece catalogada como subdisciplina (2512.01) perteneciente a la disciplina Ciencias del Espacio (2512), incluida a su vez en el campo Ciencias de la Tierra y el Espacio (25). 


\subsubsection{La Astrobiología como actividad científica}

Es frecuente en la literatura astrobiológica afirmar que la Astrobiología es una actividad que precisa del concurso de numerosos campos, disciplinas y subdisciplinas científicas: astronomía y astrofísica — cosmología, planetología, radioastronomía...-, física, química, biología, geología, ecología, antropología, informática, tecnologías de la exploración espacial, ciencias de la información, etc. Por esta razón, es un lugar común afirmar que la Astrobiología posee un carácter multi o inter-disciplinar.

Las investigaciones astrobiológicas precisan tal cantidad de recursos económicos, humanos y tecnológicos, y de un nivel tan especializado y cualificado, que ningún país puede afrontarlas en solitario. Esta característica de la Astrobiología, advertida ya por Lederberg en su varias veces citado escrito seminal, obligó a la NASA a buscar la colaboración internacional con gobiernos y agencias espaciales de otros países para llevar adelante programas y proyectos de investigación y exploración espacial y planetaria, también de carácter astrobiológico. Este aspecto sitúa a la Astrobiología en la tradición de la Big Science - cuyo origen se halla en el Proyecto Manhattan, con la construcción de la Bomba Atómica-, y comporta aspectos de interés filosófico, como las relaciones de la Astrobiología con los objetivos de los poderes político, militar y económico; su coincidencia o no con los de la ciudadanía y la sociedad en general; si son éstas cuestiones que debieran interesar a los científicos que encuentran en la nueva disciplina una oportunidad profesional; si resultan socialmente justificables tales inversiones - vg., en forma de retornos civiles- o hay necesidades más urgentes que atender; la implementación de programas y estrategias para la creación de una opinión social favorable a la nueva disciplina, y la utilización de los mass media para su difusión, buscando disminuir posibles resistencias a la aceptación de sus múltiples y diversas necesidades y exigencias, etc.

Más allá de estos aspectos sociales, éticos y políticos, algunos autores destacan que la colaboración entre ciencias que demanda la Astrobiología está incidiendo en una nueva forma de hacer y entender la ciencia - la transdisciplinariedad-, diferente de la multi e inter-disciplinariedad ${ }^{21}$. ¿Es realmente la Astrobiología el motor de una nueva revolución científica?

21 Tal es el caso, vg., de Rodríguez-Manfredi: «la Astrobiología va más allá. Esta nueva ciencia supera los límites de la interdisciplinariedad (...), instalándose en el dominio de la transdisciplinariedad. En otras palabras, la Astrobiología se nutre de la sinergia existente entre las distintas disciplinas que aúnan sus esfuerzos en esta área, creando en muchas ocasiones sus propios métodos, sus normas y sus objetivos. La transdisciplinariedad no sólo supone un mero "intercambio" de información entre los representantes de las diversas disciplinas participantes. Va más allá, implicando a estos participantes en el aprendizaje de un lenguaje común, y en la adquisición de unos nuevos esquemas y planteamientos mentales, inicialmente ajenos a las propias disciplinas a las que pertenecemos. Así, la transdisciplinariedad ligada a la Astrobiología genera nuevos horizontes a explorar en el marco del avance científico. En esa línea, la transdisciplinariedad encuentra su máxima expresión en esta nueva área 
La literatura astrobiológica insiste igualmente en el papel de la Astrobiología como área paradigmática de aproximación y colaboración no sólo entre las ciencias naturales, sino también entre éstas y las humanidades. Estos aspectos metodológicos y de interacción entre saberes, personas y colectivos, así como sus consecuencias a la hora de hacer y entender la ciencia, son cuestiones meta-científicas que interesan a la filosofía, además de otras ciencias sociales.

En definitiva, la Astrobiología como actividad científica presenta aspectos que interesan, cuando menos, a la Filosofía de la Ciencia, la Epistemología, la Lógica (Metodología y Método Científico), la Sociología de la Ciencia, la Ética —especialmente Ética de la Ciencia—, la Filosofía de la Técnica, la Filosofía Política y la Filosofía Social.

\subsubsection{La Astrobiología como estudio científico de la vida}

De un modo general, las cuestiones básicas que interesan a la Astrobiología son: cómo se origina y evoluciona la vida, su posible existencia en algún otro lugar del universo y cuál será su futuro, tanto en nuestro planeta como fuera de él (cf. NASA Astrobiology Roadmap).

La vida constituye, por tanto, el objeto de estudio de la Astrobiología. Pero, tradicionalmente, el estudio de la vida ha sido tarea de la biología, por lo que la Astrobiología deberá justificar su especificidad frente a otras disciplinas biológicas o desaparecer por innecesaria.

Sin embargo, frente a la biología, la Astrobiología ofrece una visión más amplia e integrada del fenómeno de la vida, particularmente con la astronomía y la astrofísica, así como con las tecnología espaciales, contextualizándolo cósmicamente, y esto en un triple sentido: i) conectando el origen de la vida en la Tierra con el entorno cósmico, ii) interesándose por la posible existencia y distribución de vida en otros lugares del universo, y iii) preguntándose por el futuro de la vida en el universo.

Con su proceder, la Astrobiología sitúa a la biología y las demás ciencias de las que se sirve en un nuevo nivel, inédito hasta el presente, obligándolas a redimensionar y revisar sus conceptos, teorías, presupuestos, métodos y técnicas, fundando así un área especifica de interés ${ }^{22}$.

Al igual que la biología, la Astrobiología se interesa por el origen y evolución de la vida, por los procesos que tienen lugar desde el nivel molecular hasta los

del conocimiento, dado el número y naturaleza de las disciplinas que tienen cabida en ella: desde las más intuitivamente relacionadas como son la química, la biología, la geología, la física, la ingeniería, la ciencia de la computación o la astronomía/astrofísica, a otras que también tienen interesantes aportaciones que realizar, como la filosofía, la sociología o la ética.» (Rodríguez-Manfredi, J. A., «La innovación tecnológica en la astrobiología como motor de cambio en la mentalidad actual», Letras de Deusto, no 118, vol. 38 (enero-marzo 2008), p. 70).

22 Cf. vg. NASA Astrobiology Roadmap, 2008: «El amplio carácter interdisciplinar de la Astrobiología nos obliga a esforzarnos por lograr una comprensión más amplia e incluyente de los fenómenos biológicos, planetarios y cósmicos». 
ecosistemas, tomando para ello la selección natural (Darwin) como base explicativa del dinamismo evolutivo. Pero la Astrobiología lo hace, además, desde una perspectiva diferente, que describimos a continuación ${ }^{23}$ :

a) Como la biología, la Astrobiología se ocupa del origen de la vida, es decir, de la materia y su organización en sistemas vivos, pero interesándose por la conexión cósmica de su objeto de estudio, esto es, por descubrir y comprender los procesos que tienen lugar entre el origen y formación de los cuerpos planetarios y la evolución prebiótica, ampliando así el interés de la biología por los procesos moleculares que posibilitan la transición entre la materia inerte y la viviente en la Tierra.

b) En segundo lugar, la Astrobiología se interesa por el fenómeno de la pluricelularidad identificando, como la biología, los factores biológicos e influencias ambientales terrestres que intervienen en su origen y desarrollo. Pero la Astrobiología amplía dicho estudio a los posibles factores e influencias cósmicas y procesos planetarios implicados, lo que hace esta nueva disciplina se interese también por la distribución de vida compleja en el Universo.

c) La Astrobiología investiga la evolución temprana de la vida y la biosfera para comprender la naturaleza de los organismos más primitivos, así como los entornos en los que éstos evolucionan. Se sirve para ello de los registros molecular — seres vivos- y geológico — rocas y meteoritos-, que conservan la historia evolutiva del planeta y de los seres que se han desarrollado en él.

Pero los organismos vivientes son sistemas más complejos que los físicos, y su organización depende de un programa - código genéticoque almacena y transmite información mediante moléculas. Por ello, la Astrobiología se interesa también por el origen de la primera molécula autoreplicante - mundo RNA, DNA - que dio paso a la primera célula con información heredable. A diferencia de la biología, el interés de la Astrobiología por estas cuestiones lo es en función de su conexión con factores cósmicos y planetarios (exo), así como la posibilidad de que tales procesos también hayan acontecido en algún otro entorno distinto del terrestre.

d) La Astrobiología, además de interesarse por las condiciones planetarias y cósmicas - galácticas-implicadas en el origen de la vida, tales como la formación y estabilidad de planetas habitables, también lo hace por la formación de moléculas orgánicas complejas en el espacio y su transporte hasta las superficies planetarias.

23 Para establecer las diferencias que siguen tomamos básicamente como referencia las líneas que establece NASA en la elaboración del nuevo Astrobiology Roadmap, recogidas por Gronstal, A. L., en «The next steps for Astrobiology's Roadmap», Astrobiology Magazine $(17 / 06 / 2013)$. 
Lo expuesto muestra que la Astrobiología integra necesariamente cuestiones básicas de la biología que ya son objeto de reflexión por parte de la Filosofía de la Biología, una joven rama de la Filosofía constituida, no sin dificultades, como subdisciplina de la Filosofía de la Ciencia —código UNESCO 7205.01en los años 80; pero también la aportación fundamental de la Astrobiología: el énfasis en la conexión cósmica de la vida, es decir, en tanto que fenómeno a considerar sub specie Universorum. Este aspecto, que a nuestro juicio constituye una de las mayores contribuciones científicas de la historia y marca un hito cultural, abre el debate acerca de la legitimidad de la Filosofía de la Astrobiología para aspirar al estatus de subdisciplina filosófica con entidad propia, a la altura de la Filosofía de la Biología, antes que al de subapartado de ésta. Obviamente, nosotros ya hemos optado (véase nuestra propuesta de definición), y argumentaremos nuestra posición al final del escrito, en el epígrafe dedicado a conclusiones.

Por el momento, digamos que la elaboración de una Filosofía de la Astrobiología, y su consiguiente reconocimiento académico, pasará por justificar su especificidad frente a la Filosofía de la Biología ya existente, lo que sólo sucederá si en su reflexión deja traslucir lo peculiar del nuevo enfoque astrobiológico. Si la Astrobiología no es una disciplina innecesaria, tampoco lo será la meta-reflexión acerca de ella, es decir, de su sujeto de estudio y su actividad (objetivos, estrategias, resultados, consecuencias, implicaciones...).

Como sucede con otras ciencias particulares y su correspondiente reflexión filosófica, será tarea de una FAB la clarificación de principios, términos y conceptos empleados por los científicos en sus teorías, evitando confusiones terminológicas y ayudando a éstos en la elaboración de definiciones ${ }^{24}$.

En este sentido, incumbe a la FAB contribuir a una adecuada definición de vida. Si ya la Biología y la Filosofía de la Biología encontraban dificultades en ello, la nueva perspectiva cósmica aportada por la Astrobiología vuelve más complejo el problema, en especial al abrir la cuestión del origen de la vida a nuevas posibilidades: ¿Procede la vida en la Tierra del espacio - panspermiao es el resultado de procesos autónomos acontecidos en nuestro planeta? ¿Podrían haberse repetido tales procesos en otros lugares del Universo (segunda génesis)? ¿Ha habido más de un origen de la vida en la Tierra y, de ser así, cuál habría sido la ventaja adaptativa que permitió sobrevivir a nuestro ancestro común? ¿Cabría la posibilidad de una biota en la sombra, prueba de una segunda génesis exitosa en nuestro propio planeta? ¿Podría la vida evolucionar en ambientes extraterrestres? ¿De qué modo? ¿Qué caminos podría seguir la evolución en otros lugares del universo? ¿Cuán diferentes podrían ser las formas de vida extraterrestres? ¿Podrían diferir tanto de la nuestra que, en caso de topar con ella, nos fuera imposible reconocerla? ¿Cómo afecta esto a su búsqueda?

24 Nos servimos del artículo de Antonio Diéguez: «Qué es la filosofía de la biología?», Encuentros en la biología (enero-febrero 2011) Vol. 4, no 132, pp. 3-5, como referencia para identificar algunos temas y aspectos la Filosofía de la Biología y compararlos con los de una Filosofía de la Astrobiología, dentro siempre de los límites del presente escrito. 
El problema se agrava ante los avances en vida artificial y vida sintética: ¿Cuál es el sistema vivo más simple posible?

Ante lo apuntado arriba, parece evidente que, además de para la Filosofía de la Biología, también será relevante para una FAB la discusión —aclaración y delimitación- de conceptos fundamentales para la Astrobiología, tales como: gen, procariote, eucariota, fitness, función, adaptación, selección natural, oportunidad, mecanismo, complejidad, emergentismo, información, nicho ecológico, biodiversidad, biocentrismo, equilibrio puntuado, evolución convergente, progenote o ancestro común, extinción, catastrofismo...

Es igualmente relevante para una FAB reflexionar sobre la relación entre diversas nociones, como las de evolución y progreso, revisar los niveles sobre los que actúa la selección natural o atender a los diferentes aspectos y discusiones que se suscitan al abordar científicamente el problema del origen de la vida, tales como las aproximaciones top-down/bottom-up, el debate abierto por el Mundo RNA, o la controversia entre los partidarios de la replicación primordial y los del metabolismo primigenio y el acercamiento a la biología de sistemas y la vida sintética surgido de la síntesis de ambas posturas, entre otros.

Más aún, es posible encontrar en la literatura astrobiológica discusiones que cuestionan el paradigma explicativo estandar sobre el origen de la vida - vida como resultado de la organización de la materia y sus leyes-, proponiendo un giro copernicano en el enfoque del problema al concebir un universo material regido por leyes y dinámicas biológicas (Gardner). Tal sugerencia, a pesar de su carácter especulativo, presenta por su forma y contenido importantes implicaciones y consecuencias con resabios hegelianos y profundo alcance filosófico, e incluso teológico.

Entre los aspectos y conceptos que interesan a la FAB se hallan aquellos que surgen o se actualizan a la luz de la transdisciplinariedad característica de la actividad astrobiológica — particularmente en la interacción con las ciencias espaciales-, y siempre desde su particular enfoque cósmico. Así, por ejemplo, corresponde a la Astrobiología caracterizar, a partir de los conocimientos sobre la vida en la Tierra, los ecosistemas que podrían existir en entornos extraterrestre - extremos o de tipo terrestre- a fin de establecer los biomarcadores que permitan a los biosensores de las sondas exploradoras enviadas a otros mundos reconocer la posible existencia de vida en éstos. En este contexto es frecuente el empleo de términos con una mayor carga o especificidad astrobiológica, tales como: biogeocentrismo, biomarcadores, biofirmas, extremofilia, archea, biosensores, bioinformática, vida sintética, habitabilidad, zona de habitabilidad, rango de la vida, biota en la sombra, bombardeo temprano, esfera de observación, esfera de inteligencia, metalicidad..., términos todos ellos que precisan atención filosófica.

La Filosofía de la Astrobiología se interesa también por los aspectos filosóficos que afectan al carácter explicativo de las ciencias en general, incluida la biología, tales como las nociones de causalidad, azar y necesidad, pero que la Astrobiología obliga a revisar, así como su papel y peso en el mecanismo evolutivo — selección natural—, en función de su particular enfoque cósmico. 
Por ello, es especialmente relevante para la Astrobiología la constatación del fenómeno de la evolución convergente, dadas sus implicaciones para el azar en los sistemas complejos - tanto bioquímicos como astrofísicos-, así como en los distintos niveles evolutivos, desde el molecular hasta la inteligencia (cerebros). En consecuencia, interesará a la FAB el debate sobre la cientificidad de las explicaciones funcionales o teleológicas, deslindando lo que es ciencia de lo que no (vg. creacionismo y diseño inteligente), así como el problema de los reduccionismos explicativos, siempre desde la particularidad que impone el enfoque astrobiológico que venimos señalando.

En esa tarea de clarificación de conceptos y términos la filosofía no sólo aporta lógica, sino también capacidad hermenéutica. A la filosofía le interesa el modo en que el científico lleva a cabo su tarea en su esfuerzo por obtener explicaciones de los fenómenos (metodología), el enfoque elegido y los presupuestos epistémicos, culturales, históricos y sociales subyacentes, que ayudan a comprender mejor el valor y adecuación tanto de su tarea como de las herramientas conceptuales empleadas. Todas ellas son cuestiones abordadas en la literatura astrobiológica.

La Filosofía de la Astrobiología está obligada, igualmente, a considerar el estatuto científico de la Astrobiología, así como la estructura lógica y la contrastabilidad de sus hipótesis: ¿Es científica la hipótesis Existe Vida Extraterrestre, teniendo en cuenta su no falsabilidad? Por el contrario, la hipótesis No Existe Vida Extraterrestre no es verificable debido al problema de la inducción, pero sí resulta en cambio falsable dado que parece factible la comprobación experimental de la evolución de microorganismos en el sistema solar. Analizar la estructura y validez de los métodos y la contrastabilidad de las hipótesis son tareas filosóficas.

Es claro, por tanto, que la filosofía está llamada a mostrar la estructura conceptual de la Astrobiología y su especificidad frente a otras disciplinas o ciencias, especialmente la biología, así como analizar los argumentos y criterios que fundamentan tal deslinde.

En definitiva, la Astrobiología genera nuevos y enriquecedores enfoques de temas y problemas antiguos que constituyen la esencia misma del pensamiento occidental desde sus orígenes, tales como el determinismo, el azar, o la existencia de sentido, racionalidad o propósito en los procesos de transformación, sean éstos vivientes o físicos. La Astrobiología también actualiza teorías, temas y problemas biológicos y filosóficos a la luz de los nuevos datos y conocimientos por ella obtenidos, poniendo de este modo las bases para un planteamiento científico de temas y problemas que hasta la fecha parecían inevitablemente especulativos o metafísicos. Y en cualquier caso, es evidente que la sola existencia de la Astrobiología plantea ya una cuestión de inmenso calado filosófico: ¿es la vida en la Tierra una excepción o el episodio local de una tendencia o necesidad inserta en la base material del Universo (imperativo cósmico)?

Las cuestiones apuntadas en este epígrafe son de la incumbencia, cuando menos, de la Filosofía de la Ciencia, la Lógica, la Filosofía de la Biología, la 
Filosofía de la Naturaleza, la Filosofía de la Vida, la Filosofía de la Materia, la Epistemología, la Ontología y la Hermenéutica.

\subsubsection{Astrobiología y vida humana: inteligencia y psiquismo}

Al reconsiderar el fenómeno de la vida desde su peculiar enfoque o perspectiva cósmica —sub specie Universorum - la Astrobiología obliga también a un replanteamiento de la pregunta científica por la vida humana —su comienzo, evolución y posición en el árbol de la vida-, a la vez que renueva la reflexión filosófica sobre el puesto del ser humano en el Cosmos y, en general, sobre el sentido de su existencia. Tales son los aspectos implícitos en la pregunta: ¿Estamos solos en el Universo?

Dentro de este contexto, la literatura astrobiológica suele centrar su interés en el fenómeno de la inteligencia (cerebro) — su aparición y evolución-, especialmente la humana. En este marco, la discusión sobre la existencia o no de coincidencias notables en las constantes fundamentales de la física y la bioquímica conspirando a favor de la vida se extienden al surgimiento de la inteligencia: ¿existen tendencias en los diferentes niveles de complejidad y evolución desde el astrofísico y bioquímico al psíquico; desde el polvo estelar a cerebros? Así, son objeto de discusión en este contexto conceptos como ajuste fino (fine-tuning), su relación con el Principio Antrópico y sus diferentes versiones, o la compatibilidad entre los conceptos de adaptación radiativa (Darwin), convergencia evolutiva y versión débil del Principio Antrópico: ¿Qué se conservaría en una nueva evolución? ¿Volvería a surgir una inteligencia como la humana en una segunda evolución? ¿Qué tipo de corporalidad es necesaria para la existencia de inteligencias, en especial las altamente desarrolladas: qué tipo de cuerpos, sensorios y cerebros, más aún ante la existencia de máquinas que «piensan»? ¿Y la inteligencia artificial? La neurociencia, la física cuántica, la biología y la ingeniería tienen mucho que aportar en este campo y a la reflexión filosófica aparejada. Términos clásicos como cyborg (Clynes y Kline) recobran actualizados su significado original —adaptación de humanos a ambientes extraterrestres-, y surgen otros nuevos igualmente desafiantes, como el de entidades post-biológicas (Dick), todos ellos apuntando hacia el trans-humanismo como horizonte antropológico.

Implicadas con todas estas cuestiones se hallan otras con larga tradición filosófica, pero que adquieren una nueva dimensión desde la Astrobiología, tales como la especificidad del ser humano — qué nos hace diferentes de otras especies-, el funcionamiento del psiquismo, la universalidad del pensamiento formal, de la lógica, de la matemática o los aspectos evolutivos de la inteligencia y el psiquismo, incluidas sus implicaciones para la constitución y fundamentación de una vida moral, así como de la cultura (proceso de humanización). Cabe preguntarse, incluso, si tiene sentido hacerse tales preguntas. La especulación puede ayudar a entender el sentido, implicaciones y profundidad de las mismas. 
A su vez, los estudios sobre otro tipo de cerebros e inteligencias surgidos en nuestro planeta, tales como los de mamíferos y grandes simios, y especialmente los evolucionados en medios distintos del humano, como el acuático —cefalópodos y cetáceos-, pueden ayudar a comprender la interacción medioneurona y su influencia en el surgimiento y evolución de sistemas nerviosos, particularmente los complejos. En cualquier caso, la Astrobiología considera que existe la posibilidad de que muchas de esas cuestiones puedan ser resueltas experimentalmente. Así, por ejemplo, la constatación de convergencia en los diferentes niveles evolutivos posibilitaría la predecibilidad, permitiendo la fijación y selección de biomarcadores adecuados para misiones exploratorias sobre el terreno en otros lugares del universo, o bien a distancia mediante radioastronomía. Para estas discusiones son también relevantes conceptos y aspectos como: nicho de inteligencia, esfera de observación, metalicidad, antigüedad del universo, biomarcadores...

Lo expuesto en este apartado interesa, cuando menos, a la Filosofía del Conocimiento, la Filosofía de la Mente, la Filosofía de la Naturaleza, la Filosofía de la Tecnología y la Antropología Filosófica.

\subsubsection{Astrobiología, realidad y conocimiento}

Sensorios, sistemas nerviosos, cerebros, percepciones... Todo ello implica aspectos relativos a la forma en que los distintos individuos y las diferentes especies representan — sienten, organizan, viven, comprenden, categorizan...lo real. La Astrobiología replantea nuevamente una cuestión filosófica central: ¿Qué es la realidad y cómo la conocemos?, y ello en un triple sentido, al menos:

a) Ligado a las cuestiones apuntadas en el apartado anterior: ¿cómo percibirán la — su - realidad otras formas de vida, otras inteligencias y psiquismos evolucionados en entornos cósmicos diferentes, en particular si se tratara de seres altamente evolucionados, incluso cultural y técnicamente?

b) En un sentido menos especulativo: la Astrobiología emplea tecnologías y ciencias del s. xx que permiten una aproximación o representación de la realidad distinta de la tradicional. Sabido es que las tecnologías empleadas por la Astrobiología, como sucede con cualquier otra ciencia, interactúan con lo real y con nuestros sentidos-intelecto. Analizar las causas y el modo como esto sucede y sus consecuencias para nuestra forma de percibir y comprender el mundo y a nosotros mismos es también una tarea filosófica. Es preciso advertir y reflexionar, por tanto, sobre el modo y grado en que la Astrobiología pueda estar contribuyendo no sólo a un cambio de mentalidad colectiva, al menos en la cultura occidental, sino también, y sobre todo, a un nuevo modo de ser y concebir la racionalidad, lo real y a nosotros mismos. Algunos autores consideran que la Astrobiología — su actividad y técnicas- está generando un cambio de teoría de tal calado que desembocará en una revolución 
del paradigma científico dominante en la actualidad —-biogeocentrista-, con la consiguiente reelaboración de nuestra imagen de lo real, del mundo, de nosotros mismos y de nuestra posición y responsabilidad en el Universo. Así lo ha entendido vg. Mayz Vallenilla, cuando analiza la Astrobiología a la luz de su teoría de la Meta-técnica ${ }^{25}$.

3) Preguntas como: ¿Qué es la realidad? ¿Tiene ésta algún sentido? ¿Qué relación hay entre el caos y el orden? ¿Es inteligible la naturaleza? ¿Puede el ser humano llegar a comprenderla? ¿Es racional lo real o sólo aparentemente?, se hallan en el núcleo del pensamiento occidental. La Astrobiología incide sobre el problema de la estructura e inteligibilidad de la naturaleza al profundizar en el conocimiento del fenómeno de la vida intentando desentrañar su conexión cósmica, a la vez que buscando un segundo caso de vida en el universo cuyo hallazgo validaría ciertas líneas de pensamiento acerca de la existencia de tendencias en el cosmos, debilitando otras.

Todos estos aspectos aludidos son incumbencia, cuando menos, de la Filosofía del Conocimiento (Teoría del Concepto, Teoría de la Percepción y Teoría de la Razón...), así como de la Filosofía Social (Filosofía de la Cultura, Filosofía de la Historia....). Igualmente lo es de la Epistemología y la Gnoseología, la Ontología, La Filosofía de la Naturaleza, la Filosofía de la Técnica y la Antropología Filosófica (la Hermenéutica en particular).

25 Mayz Vallenilla hace un análisis epistemológico crítico de la ciencia astrobiológica desde el particular enfoque de la meta-técnica, situándolo en el contexto más amplio de una crítica epistémica que desborda el marco estrictamente astrobiológico, dado que afecta a los fundamentos mismos de la ciencia y de la técnica en cuanto tales. La radicalidad de dicho planteamiento, así como lo revolucionario del concepto central que le sirve de base, la meta-técnica, quedan de manifiesto al advertir que tal crítica implica, en palabras del propio autor, la «superación de la Razón y la racionalidad que informan y sostienen a la técnica y/o al conocimiento tecno-científico tradicional». Desde la perspectiva apuntada, el Dr. Mayz Vallenilla nos propone atender al logos, o fundamento racional, de la Astrobiología, a partir del cual se forjan sus nociones y conceptos fundamentales como ciencia. En su tarea de des-velamiento, el profesor Mayz Vallenilla observa que la astrobiología se erige sobre un «logos óptico-lumínico», de carácter antropomórfico, antropocéntrico y geocéntrico que, sin embargo, y al igual que sucede con otras ciencias, como la astrofísica, ha sido superado ya por los artefactos de carácter meta-técnico, es decir, trans-racionales y trans-humanos (descentrados de lo humano y de lo terrestre), que ella misma emplea habitualmente para su labor investigadora. Así, pues, la propia razón y racionalidad naturales del hombre habrían creado un nuevo tipo de razón y racionalidad fundamentadas en un logos de nuevo cuño, el logos meta-técnico, que instituye un mundo nuevo (lenguaje, técnica, relaciones, instituciones, conceptos....) y una nueva forma de ordenarlo (sintaxis), acorde con un nuevo tipo de ser humano que se nota encerrado en, y lucha por liberarse de, los límites congénitos de su cuerpo y mente naturales (Cf. Mayz Vallenilda, E., Fundamentos de la Meta-Técnica, Barcelona: Gedisa, 1993, y "Astrofísica y Meta-Técnica», en «Astrobiología y Filosofía», Letras de Deusto (2003) Vol. 33, no 98, pp. 217-224). 


\subsubsection{Astrobiología y comunicación social de la ciencia y la tecnología}

Existen significativas interacciones de la Astrobiología con la ciencia ficción, así como con el imaginario y la cultura popular. Hay autores que atribuyen el tirón y carácter movilizador de la Astrobiología en las sociedades occidentales al halo re-sacralizador que le comunicarían sus concomitancias con el misterio del universo y la existencia humana, fácilmente manipulable -incluso para fines sectarios. Por otra parte, el hecho de que la Astrobiología sea una disciplina a cuyo nacimiento, estructuración, institucionalización y expansión estamos asistiendo nos concede una posición privilegiada para observar esa relación y comprender la propia naturaleza de la ciencia y sus relaciones con el poder, la política o la economía, así como los juegos de intereses movilizadores y amenazas tanto entre las diversas disciplinas científicas implicadas con la Astrobiología como entre sus actores. En un epígrafe anterior hacíamos referencia a la elaboración y puesta en marcha - cuando no, orquestación- de programas y estrategias implicando a los mass media en la creación de una opinión social favorable —o menos refractaria - a la aceptación de la nueva disciplina con sus múltiples y diversas exigencias.

Todo ello es de interés, cuando menos, de la Sociología de la Ciencia y la Ética de la Ciencia.

\subsubsection{La Astrobiología y la Ética}

El interés por la posible existencia de ecosistemas en otros mundos y su búsqueda activa condiciona las perspectivas o enfoques éticos de la actividad astrobiológica. La Astrobiología, en tanto que exobiología, emplea tecnologías de exploración espacial para la detección in situ de vida en nuestro sistema solar. Más allá del mismo, tal búsqueda es actualmente inviable por razones tecnológicas. Desde el comienzo de la era espacial, con la salida de la Tierra del primer artefacto humano —Sputnik 1 (04/10/1957) —, existe el riesgo real de contaminación planetaria por microorganismos, tanto de nuestro planeta como de otros entornos. En la actualidad, el peligro es mayor con el retorno de muestras recogidas en otros cuerpos celestes, como la Luna, cometas o asteroides. La posibilidad de contaminación planetaria es una consecuencia consustancial a las actividades espaciales que obliga a numerosas acciones preventivas y, en caso de producirse, a su control. Existen en la actualidad protocolos de protección biológica o planetaria para las naves y misiones espaciales (COSPAR). Todos ellos son aspectos que incumben a la reflexión ética, particularmente la Ética ambiental.

Pero la Astrobiología también desarrolla su actividad en nuestro planeta. En este sentido, existen tres aspectos de la Astrobiología que deben interesar a la ética desde la perspectiva anteriormente apuntada: la búsqueda y estudio de microorganismos adaptados a condiciones extremas, ii) las investigaciones sobre vida sintética (biología sintética) y, iii) la detección de una posible biosfera en la sombra. 
Un grupo diferente de aspectos éticos es el suscitado por la cada vez más cercana posibilidad de colonización de mundos próximos y su terraformación.

Hay otros aspectos éticos implicados con la Astrobiología, aunque más especulativos por el momento, como la experimentación con otras posibles formas de vida que pudieran hallarse, o su utilización para diversos fines - comerciales, médicos...-, especialmente en el caso de no ser microbianas sino pluricelulares sintientes e, incluso, con cierto grado de inteligencia. Con la Astrobiología, la ética adquiere una dimensión cósmica, y algunos autores hablan ya de una ética cosmocéntrica ${ }^{26}$.

Aún más especulativas resultan por ahora, aunque insoslayables, las cuestiones éticas relativas a una posible detección de formas de vida dotadas de sentimientos e intelecto con capacidad de formalización y simbolización productoras de cultura y civilización, lo que obligaría a revisar algunos conceptos éticos centrales, como el de persona. Estas son cuestiones generadas principalmente ante la posibilidad abierta por el discutido programa astrobiológico complementario de búsqueda radio-astronómica de inteligencias extraterrestres tecnológicamente avanzadas SETI, del que nos ocuparemos más abajo.

Sea como fuere, es claro que en ambos casos, trátese de formas de vida superiores como inferiores, surge el debate ético entre biocentrismo y antropocentrismo acerca del origen de los valores, si bien redimensionado desde el enfoque cósmico que le aporta la Astrobiología. Es igualmente pertinente el debate moral y ético que suscita la propia actividad astrobiológica, considerando su origen, el tipo de investigaciones que realiza, los objetivos que le interesan y la ingente cantidad de recursos humanos y económicos que todo ello precisa, especialmente ante los acuciantes y variados problemas a los que se enfrenta el genero humano en la actualidad.

Todos estos aspectos indicados involucran diversos aspectos de la Ética, como la Ética ambiental, Ética de la Ciencia, Axiología o Códigos de Conducta Ética y de Valores.

\subsubsection{Aspectos filosófico-teológicos de la Astrobiología}

Algunos temas de interés filosófico-teológico (Teología Natural) que plantea la Astrobiología son los siguientes:

- La relación entre el ser y el pensar desde la perspectiva de la racionalidad humana y el poder creador de Dios, esto es, la tendencia de la lógica humana a imponer su discurso - reduccionismo o determinismo logicista- a las posibles formas del ser que hayan podido crear la libertad y omnipotencia divinas (Pérez de Laborda);

- La necesidad de proponer nuevas maneras de entender el ser de Dios ante la posibilidad de que haya otros mundos habitados por seres

26 Lupisella, M. y Logsdon, J., «Do we need a Cosmocentric Ethic?», Congreso de la Federación Internacional de Astronáutica (IAF), 1997. 
altamente inteligentes poseedores de cultura: nuevo ecumenismo de corte cósmico (Miret Magdalena) o la necesidad de una Cosmoteología (Dick);

- Los diferentes modos de abordar el problema de la vida extraterrestre en las distintas religiones y teologías;

- El impacto en las religiones y en sus Iglesias de un eventual hallazgo de vida extraterrestre, como la posible revisión del concepto de persona desde una perspectiva religiosa - vg. como apertura consciente al otro (Buenaventura)—, con independencia de sus peculiaridades morfológicas y/o psíquicas; o la incidencia en el dogma cristiano de la salvación - universalidad de la encarnación y efecto redentor de la figura de Cristo (cristocentrismo) - de una hipotética existencia en otros mundos de formas de vida llamadas a la salvación y, por ello, susceptibles de redención;

- Incidencia en la relación ciencia-religión: ¿glorificaría a Dios la existencia de otros seres inteligentes o esto acabaría con las religiones?

- Revisión de conceptos como contingentismo, creacionismo, determinismo, intervención divina, milagro...;

- ¿Son interpretables desde la teología natural las nociones de fine-tuning y la posible existencia de tendencias en bioquímica como indicadoras de propósito? (Chela-Flores); análisis de las interacciones entre la concepción de la vida como imperativo cósmico, el Principio Antrópico y ciertas interpretaciones de la noción de finalidad - teleología - no desechables en biología (Ayala) por un lado, y deslinde de las mismas de la posición acientífica del Diseño Inteligente.

Todas ellas son cuestiones que incumben, cuando menos, a la Teología Natural, Sistemas Teológico-Filosóficos, Filosofía de la Religión y Ética Religiosa, así como a otras disciplinas que se interesan por el fenómeno religioso desde diferentes perspectivas, tales como la Antropología, particularmente la Antropología Cultural y de la Religión y la Historia de las Religiones.

\subsubsection{Busqueda complementaria SETI: aspectos filosóficos}

SETI (Search for ExtraTerrestrial Inteligence), es un programa de rastreo del cielo mediante potentes radiotelescopios —radioastronómico- en busca de señales que evidencien la existencia de civilizaciones tecnológicamente avanzadas. Su origen estuvo en el Proyecto Ozma, puesto en marcha por el astrónomo norteamericano F. Drake en 1960. El programa SETI, que en cierto momento llegó a ser financiado por la NASA, es sostenido actualmente con fondos privados y posee el estatus de programa astrobiológico complementario, contando con el apoyo estratégico del NASA Astrobiology Institute (NAI).

Mientras los astrobiólogos pertenecientes a disciplinas de las ciencias de la vida y de la Tierra, como biólogos y geólogos, se interesan por las formas de vida elementales y su evolución —enfoque de abajo hacia arriba—, los astrónomos 
de SETI escudriñan los cielos en busca de una señal de origen artificial no humano - enfoque de arriba hacia abajo- que no llega, pero que de suceder tendría inmensas implicaciones para todos los sectores de la ciencia y la cultura; mayores que las derivadas de un éxito de los astrobiólogos del enfoque complementario opuesto

La propia existencia de SETI es ya en sí misma una cuestión para el debate filosófico y antropológico por diversos motivos, como las discusiones acerca de la validez de los presupuestos y fundamentos filosóficos, metodológicos y tecnológicos que sostienen y dan sentido a su labor - entre ellos la famosa y controvertida Ecuación de Drake - a la vez que justifican los objetivos e inversiones del programa, o las motivaciones antropológicas profundas con las que dicho programa conecta, haciéndolo tan atractivo para unos como denostado por otros.

SETI es, en cualquier caso, el programa astrobiológico que más directamente enlaza con la tradición filosófica sobre la pluralidad de mundos habitados, puesto que los pensadores occidentales consideraron desde el principio esta cuestión desde una perspectiva antropocéntrica - la existencia de seres dotados de inteligencia de tipo humano-, o incluso zoocéntrica, pero nunca microbiana. El descubrimiento de formas de vida microscópicas data de mediados del siglo XVII y la microbiología no se asienta como ciencia hasta mediado el XIX. El descubrimiento de los microorganismos y su progresivo conocimiento posibilitó una nueva forma de imaginar la existencia de vida extraterrestre. El comienzo de la era espacial (1957) permitió concebir como factible su búsqueda científica en otros mundos. Tal sucedió, como se dijo oportunamente, en el origen del programa exobiológico iniciado por la NASA a finales de la década de los años cincuenta del siglo pasado. Hay, pues, una interesante relación a explorar por la filosofía entre conocimiento, imaginación, ciencia, tecnología y sociedad.

El proyecto con el que se estrenó SETI fue Ozma (1960), consistente en un rastreo del cielo en busca de señales (radioescucha o SETI pasivo).

Pero también las naves espaciales enviadas a otros mundos por el hombre son una clara señal de vida inteligente tecnológicamente avanzada para unas posibles inteligencias que dieran con ellas ¿Por qué no saludar y presentarnos a nuestros vecinos cósmicos? Guiados por esta idea, C. Sagan, su esposa y F. Drake dieron un paso más en el terreno de la comunicación interestelar con la elaboración y el envío de un mensaje grabado en unas placas que se colocaron en las naves Pioneer 10 y 11 lanzadas en 1972 y 1973, respectivamente.

En 1974, Drake fue más allá al codificar un mensaje que lanzó al espacio SETI activo- en forma de ondas electromagnéticas desde el radiotelescopio de Arecibo, con motivo de su reinauguración. Esta acción individual de Drake suscitó las críticas de miembros de la comunidad científica, que la calificaron de irresponsable al considerar que el mensaje podía revelar información sensible sobre la humanidad susceptible de ser utilizada contra ella por una civilización hostil. La iniciativa de Drake habría violado el acuerdo alcanzado por los científicos participantes en la Conferencia Internacional sobre Comunicación con 
Inteligencias Extraterrestres (CETI) —Byurakan, Armenia soviética, 1971-, que otorgaba a la cuestión de la comunicación interestelar estatus de interés colectivo -internacional - y aplicaba el principio de precaución al decidir mantener en el anonimato a la Tierra y a la humanidad ante nuestra ignorancia acerca del tipo de intenciones que pudieran albergar otras posibles inteligencias tecnológicamente avanzadas en el universo.

En cualquier caso, las acciones de Sagan y Drake no fueron los primeros intentos SETI-CETI de la humanidad, aunque sí los más modernos y de mayor alcance por sus fundamentos teóricos y las tecnologías empleadas, y sirvieron para evidenciar, entre otros aspectos, el interés de la composición y descifrado de mensajes interestelares para la Astrobiología, aspecto que posee numerosas implicaciones de calado filosófico y científico acerca de la naturaleza de la comunicación, del pensamiento formal, su estructura y funcionamiento, la capacidad de simbolización, la posible universalidad de la lógica, de los mecanismos de inferencia y de las matemáticas, así como las implicaciones de todo ello para una epistemología evolutiva. Pero también planteaba las cuestiones éticas y pragmáticas implicadas en las acciones individuales, así como sus repercusiones y consecuencias para el grupo a un nuevo nivel, superior incluso al planetario: el cósmico. Y lo mismo en el caso de la naturaleza y necesidad de pactos y acuerdos, así como, una vez alcanzados, sobre la conveniencia u obligatoriedad de respetarlos para la supervivencia del grupo y el progreso social, o la aplicación del principio de responsabilidad, entre otros aspectos.

La ausencia de éxito de SETI después del tiempo transcurrido y los recursos humanos, técnicos y económicos invertidos en este programa también presentan aspectos filosóficos, como el planteado por el físico Fermi cuando se pregunta: ¿dónde están? Esta cuestión, conocida como Paradoja de Fermi, incide sobre la aparente incongruencia de sostener que pueda haber inteligencias tecnológicamente avanzadas -quizá inimaginablemente avanzadas-y no haber sido halladas aún pruebas indiscutibles de su existencia, ni en forma de señales, ni de artefactos, ni de visitas. Mientras tanto, los defensores de SETI hacen frente a sus críticos adaptando sus búsquedas a los rápidos desarrollos tecnológicos y proponiendo nuevos paradigmas explicativos que permitan mantener vivas las mismas.

Los razonamientos y esfuerzos intelectuales a favor y en contra de la Paradoja de Fermi constituyen un ejercicio de lógica digno de atención, pues han generado un argumentario de sumo interés filosófico a pesar de lo especulativo del tema, o precisamente por ello. Todo este debate implica cuestiones relativas a la argumentación y la contrastabilidad — falsabilidad/verificabilidad— de las hipótesis y su cientificidad, además de obligar a la revisión y valoración de conceptos o ideas tales como: principio de mediocridad, principio antrópico, principio subantrópico, supuesto de indetectabilidad, hipótesis Rare Earth, supervivencia al éxito tecnológico, vida de una civilización, esfera de observación, edad del Universo, metalicidad... El debate involucra, igualmente, discusiones sobre aspectos de física teórica y cuántica, astrofísica y biología relativas a la 
posibilidad de los viajes en el espacio y el tiempo (entrelazamiento y coherencia cuánticos, agujeros negros, agujeros de gusano, relatividad...).

Las búsquedas SETI y las acciones CETI involucran, por tanto, en mayor o menor grado especulativo, aspectos que interesan, cuando menos, a la Filosofía del Lenguaje, diversas disciplinas y subdisciplinas de la Lógica —como Teoría de los Lenguajes Formales, Analogía, Inducción, Intuicionismo o Probabilidad-, de la Ética —Ética de individuos, Ética de Grupos...-, así como Filosofía de la Lógica, de las Matemáticas y de la Física, Filosofía de la Naturaleza - de la Materia, del Espacio y Tiempo- o Antropología Filosófica -Filosofía de la Imaginación, Filosofía de la Intersubjetividad, etc.

\subsubsection{Aspectos filosóficos de un eventual hallazgo de vida fuera de la Tierra}

No conocemos actualmente dato ni principio científico ni metafísico alguno que prohíba la posibilidad de que la vida haya surgido o evolucionado también en otros lugares del Universo, además de hacerlo en nuestro planeta. Cada vez son más los miembros de la comunidad científica que reconocen públicamente la posibilidad de la existencia de vida extraterrestre, así como de su hallazgo. La mayoría de ellos consideran que lo más razonable es suponer que tales formas de vida habrán de ser mayoritariamente microbianas, con menos probabilidad de tipo superior y menor aún inteligente o tecnológicamente avanzada. Pero teniendo en cuenta la posibilidad, y extrapolando los conocimientos actuales sobre el origen y evolución de la vida en la Tierra - el único caso conocido hasta el momento- - a otros lugares del Universo, cabe pensar que la vida en otros mundos también haya podido surgir y alcanzar diferentes grados o niveles de evolución.

En consecuencia, las implicaciones y aspectos filosóficos de su eventual hallazgo estarán en función del tipo de vida descubierta y el estado evolutivo en que ésta se halle. Dependiendo del tipo de vida encontrado, las implicaciones filosóficas serían distintas y diversas y, en consecuencia, también las disciplinas filosóficas involucradas, así como el grado en que lo estén. Lo más razonable es pensar que en otros lugares de nuestro Sistema Solar la vida no haya superado el estado microbiano, y eso es lo que busca la Astrobiología con la misiones exploradoras - sondas espaciales- sobre el terreno (búsqueda de abajo hacia arriba). Dentro de este contexto, no supondría lo mismo encontrar archeas habitando en ambientes extremos que seres pluricelulares eucariotas, pues aunque en ambos casos el hallazgo de un segundo caso de vida en el universo marcaría un hito en la historia de la ciencia y el pensamiento, al posibilitar la confirmación experimental de la universalidad de ciertas leyes o principios biológicos -el hecho de contar con un único ejemplo de vida ha situado históricamente a la biología en inferioridad respecto de otras ciencias naturales, como la física y la química-, el descubrimiento de organismos eucariotas aumentaría las probabilidades de que la vida pudiera haber evolucionado hacia la inteligencia también en otros lugares del Universo. 
En cualquier caso, el tipo de implicaciones dependería también del modo en que la vida encontrada hubiera surgido en su entorno. Tales implicaciones serían diferentes en caso de panspermia que en el de una genuina segunda génesis. La constatación de una segunda génesis permitiría confirmar la existencia de tendencias o convergencias evolutivas en la materia y la vida, pero diferentes y con distintas implicaciones filosóficas, y hasta teológicas, de las que tendría la panspermia.

Un éxito de SETI, en cambio, supondría un atajo en las búsquedas porque evidenciaría de golpe la existencia de estados evolutivos muy avanzados (enfoque de arriba hacia abajo). Un hallazgo de SETI poseería muchas más implicaciones filosóficas, y más profundas, que el hallazgo de simples microorganismos, apuntando a la existencia de tendencias organizativas o evolutivas convergentes de mayor calado y extensión en la raíz misma del universo - en la materia o polvo estelar-, que irían desplegándose en niveles evolutivos sucesivos, como el molecular y el biomolecular, no sólo hacia la vida, sino también hacia la inteligencia y el psiquismo. Esto permitiría pensar no sólo en un universo biogénico, sino también psico e, incluso, tecnogénico.

Por lo indicado, es claro que las disciplinas y subdisciplinas implicadas por el eventual descubrimiento de un segundo caso de vida en el Universo, así como el grado en que lo estuvieran, dependerían del tipo de vida hallado y de su estado evolutivo, viéndose involucradas en función de ello algunas o todas las que hemos ido indicando en el los apartados anteriores.

El descubrimiento de un segundo caso de vida en el universo evidenciaría la necesidad de abordar, y con urgencia, aspectos y temáticas que en el actual estado de la cuestión pueden parecer aplazables y hasta superfluos, pero que dado el caso devendrían súbitamente reales, pasando por tal eventualidad a formar parte de la Historia de la Ciencia y de la Filosofía y, por tanto, de los manuales escolares. De ahí que no parezca estar de más, como indicábamos al comienzo de este ensayo citando a Ortega, un ejercicio de prospectiva como el que ya realizan, dedicándole tiempo y recursos, numerosos estudiosos e instituciones (cf. Bibliografía).

\section{CONCLusiones}

A lo largo del presente trabajo hemos ido desgranando distintos temas, implicaciones y aspectos filosóficos de la Astrobiología, atendiendo tanto a su carácter de actividad científica, es decir, el estudio de la vida y su búsqueda en el universo, como al posible hallazgo de otro caso distinto fuera de nuestro planeta.

Nos hemos fijado para ello en la historia de la Astrobiología y en la literatura astrobiológica. No esperamos que nuestro análisis haya agotado los aspectos y posibilidades de una Filosofía de la Astrobiología, pero sí haber mostrado: a) que la Astrobiología es un terreno natural y fértil para la Filosofía; b) la 
posibilidad de concebir la Filosofía de la Astrobiología como un área temática y de interés bien diferenciada, tanto como lo es su sujeto de reflexión: la Astrobiología; c) la coveniencia/necesidad de sistematización de la FAB para su reconocimiento como subdisciplina filosófica específica; y d) la necesidad y urgencia de definir la FAB.

Nuestra propuesta de definición de Filosofía de la Astrobiología: «subdisciplina filosófica, perteneciente a la disciplina de la Filosofía de la Ciencia, que hace de la Astrobiología su objeto de estudio al reflexionar racionalmente sobre su condición de conocimiento, su práctica y resultados», no es neutral, pues la considera como una subdisciplina a la altura de la Filosofía de la Biología, y no una especialización de ésta. Confiamos en que después de todo lo expuesto resulten evidentes las razones para ello.

Como indicamos en su momento — notas 15 y 20—, la Astrobiología no se halla contemplada en la clasificación UNESCO de los saberes humanos ${ }^{27}$. Sí lo está, en cambio, implícitamente como Exobiología, que se encuentra catalogada como subdisciplina (2512.01) perteneciente a la disciplina Ciencias del Espacio (2512), incluida a su vez en el campo Ciencias de la Tierra y el Espacio (25). Resulta llamativo que en la clasificación UNESCO la Exobiología no se incluya en el en campo de Ciencias de la Vida (24), en el que en cambio se encuadran las diferentes disciplinas y subdisciplinas biológicas.

Por nuestra parte, hemos sostenido que es justamente el carácter exobiológico, es decir, su peculiar vinculación con las ciencias del espacio y las tecnologías de la exploración espacial (cf. epígrafe 3.2.1.3: La Astrobiología como actividad científica), y la singular y novedosa perspectiva cósmica que aporta al estudio de la vida (cf. epígrafe 3.2.1.4: La Astrobiología como estudio científico de la vida), lo que concede a la Astrobiología su especificidad frente a la biología tradicional.

Todo ello provoca que su correspondiente reflexión filosófica, si bien posee aspectos comunes con la Filosofía de la Biología, desborde sin embargo su marco de referencia no pudiendo quedar reducida, en función de dicha especificidad, del volumen que va adquiriendo tal reflexión y de la cantidad de disciplinas, subdisciplinas y perspectivas filosóficas que concita a su alrededor, a una especialidad o subgrupo de la Filosofía de la Biología, ya académicamente establecida (UNESCO 7205.01).

El mayor problema para el reconocimiento académico de la Astrobiología radica, como indicamos oportunamente, en su discutido estatus científico al no contar aún con su objeto de estudio más propio: la vida extraterrestre. Paradójicamente, la Astrobiología tiene a su favor haber hecho una de las mayores aportaciones a la historia de la ciencia y el saber humanos: consolidar científicamente la idea de que la evolución de la vida y de la inteligencia se hallan

27 Sobre este particular, cf. Martínez-Frías, J., HochBerg, D., «Classifying science and technology: two problems with the UNESCO system», Interdisciplinary Science Reviews (2007), Vol. 32, no 4, pp. 315-319. 
ligadas a la del cosmos. A partir de ahí —y es su aspecto más especulativo, por el momento-, la Astrobiología considera ambos fenómenos, la vida y la inteligencia, más comunes de lo supuesto inicialmente, habiéndose propuesto en consecuencia demostrar experimentalmente su hipótesis.

Por todo ello, sostenemos que la especificidad de la Astrobiología justifica, y precisa, su propia y específica área de reflexión filosófica que, aun presentando puntos comunes con la Filosofía de la Biología, no se confunde con ella. La Filosofía de la Biología ha tardado un siglo en alcanzar su reconocimiento académico, lo que sucedió en la década de los 80 del siglo pasado. Es posible que a la Filosofía de la Astrobiología no le lleve tanto tiempo, en parte gracias al camino avanzado por la Filosofía de la Biología. Este escrito quiere ser una contribución a ese objetivo.

\section{BibliografÍA PARA UNA FILOSOFÍA DE LA ASTROBIOLOGÍA}

Este apartado bibliográfico pretende ser una herramienta útil para la construcción de una Filosofía de la Astrobiología. No es intención del autor elaborar un listado exhaustivo de obras, sino ofrecer un punto de partida para la tarea apuntada en este escrito, a la vez que servir de prueba o apoyo a muchas de las indicaciones y afirmaciones contenidas en el mismo.

Alcibar Cuello, M., «Astrobiología, Cultura Popular y Comunicación Social de la Ciencia y la Tecnología», en "Astrobiología y Filosofía (III)», Letras de Deusto (2008) Vol. $38, \mathrm{n}^{\circ} 118$, pp. $76-85$.

Alfonseca, M., La vida en otros mundos. A la búsqueda de inteligencia extraterrestre. Madrid: McGraw-Hill, 1993.

Asimov, I., Civilizaciones extraterrestres. Barcelona: Bruguera, 1981.

AretXaga-Burgos, R., Chela-Flores, J. "Cultural Implications of the Search and Eventual Discovery of a Second Genesis» en Seckbach, J. (ed.): Genesis - In The Beginning. Precursors of Life, Chemical Models and Early Biological Evolution, Dordrecht (The Netherlands): Springer, 2012, pp. 873-890.

AretXaga-Burgos, R., "El enigma de la vida: azar y necesidad en astrobiología» en "Astrobiología y Filosofía (IV)», Letras de Deusto (enero-marzo 2012) Vol. 42, nº 134 pp. 76-105.

AretXaga-Burgos, R., «Astrobiología: entre la ciencia y la exploración», en «Astrobiología y Filosofía (III)» Letras de Deusto (2008) Vol. 38, n 118, pp. 13-27.

Aretxaga-Burgos, R., Campo, R., "Vida extraterrestre: de la especulación a su búsqueda científica», Astronomía (octubre 2006) nº 88, pp. 34-40.

Aretxaga-Burgos, R., "Astrobiología y Biocentrismo», en «Astrobiología y Filosofía (II)», Letras de Deusto (enero-marzo 2006) Vol. 36, n 110, pp: 30-36.

Aretxaga-Burgos, R., «La ciencia astrobiológica. Un nuevo reto para el humanismo del siglo XXI», en "Astrobiología y Filosofía (II)», Letras de Deusto (enero-marzo 2006) Vol. 36, n 110 , pp. 12-20. 
Aretxaga-Burgos, R., "Astrobiology and Biocentrism», en Seckbach, J., et al. (eds.): Life in the Universe. From the Miller Experiment to the Search for Life on Other Worlds, Dordrecht (the Netherlands): Kluwer Academic Publishers, 2004, pp. 345-348.

AretXaga-Burgos, R., «La ciencia astrobiológica y sus implicaciones culturales». Euskonews \& Media (2004) $\mathrm{n}^{\circ} 253$.

BAIRD, C. The Inner Limits of Outer Space. Hanover: University Press of New England, 1987.

Barrow, J. D., Tipler, F.J., The Anthropic Cosmological Principle. New York: Oxford University Press, 1988.

Barrow, J. D., Conway Morris, S., Freeland, S. J, Harper, C. (eds.), Fitness of the Cosmos from Life: Biochemistry and Fine-Tuning. New York: Cambridge University Press, 2008.

Basalla, G., Civilized Life in the Universe: Scientist on Intelligent Extraterrestrials. New York: Oxford University Press, 2006.

Bedau, M. A., Cleland, C.E., The Nature of Life: Classical and Contemporary Perspectives from Philosophy and Science. New York: Cambridge University Press, 2010.

Benner, S., Life, Universe and the Scientific Method. Gainsville: The Ffame Press, 2009.

Bennetr, J., Beyond UFOs: The Search for Extraterrestrial Life and Its Atonishing Implications for Our Future. Princeton: Princeton University Press, 2008.

Beorlegui, C., «Los retos de la Astrobiología a la Filosofía y la Teología», en «Astrobiología y Filosofía (IV)», Letras de Deusto (2012) Vol. 42, nº 134 , pp. 106-120.

BertKa, C. M. (ed.) Exploring the Origin, Extent, and Future of Life: Philosophical, Ethical and Theological Perspectives. New York: Cambridge University Press, 2009.

Billingham, J., Heins R., Milne, D., Doyle, S., Klein, M., Heilbron, J., Ashrenazi, M., MiChaud, M., Lutz, J. y shostaK, S. (eds.), Social Implications of the Detection of an Extraterrestrial Civilization. Mountain View (California): SETI Press, SETI Institute, 1994.

Billings, Linda, «Astrobiology in Culture: The Search for Extraterrestrial Life as Science», Astrobiology (octubre 2012) vol. 12, n 10, pp. 966-975.

Blumberg, B. S., «The NASA Astrobiology Institute: Early History and Organization», Astrobiology (2003), Vol. 3, n 3, pp. 463-470.

Brandstetter, T., «Life Beyond the Limits of Knowledge: Crystalline Life in the Popular Science of Desiderius Papp (1895-1993)», Astrobiology (octubre 2012) Vol. 12, n 10 , pp. 951-957.

Briones Llorente, C., «En busca de vida fuera de la Tierra: claves desde la investigación sobre el origen y la evolución de los seres vivos en nuestro planeta», en "Astrobiología y Filosofía (III)», Letras de Deusto (2008) Vol. 38, n 118, pp. 28-37.

Callicot, J. B., Beyond the Land Ethic: More Essays on Environmental Philosophy. Albany (USA): State University of New York Press, 1999.

CALlicot, J. B., "Moral considerability and extraterrestrial life», en HARGOVE, E.C. (ed), The Animal Rights/Environmental Ethics Debate. Albany (USA): State University of New York Press, 1992.

Cameron, A. G. W., Interstellar Communication. New York: Benjamin, 1963.

Самpo, R. (ed.), Vida en el Universo. Del mito a la ciencia. Santander: Fundación Anomalía, 2008.

CAmpo, R., «Astrobiología y Filosofía: cerrando el círculo original», Laguna: Revista de Filosofía (2004), no 14, pp. 185-194.

Cockell, C. S., "Astrobiology - a new opportunity for interdisciplinary thinking», Space Policy (2002), n 18, pp. 263-266. 
Cockell, C. S., "Astrobiology” and the ethics of new science», Interdisciplinary Science Reviews (2001), 26, pp. 90-96.

Chela-Flores, J., «SETI: la convergencia como un nuevo paradigma», en «Astrobiología y Filosofía (IV)», Letras de Deusto (2012) Vol. 42, nº 134, pp. 29-38.

Chela-Flores, J., The Sciencie of Astrobiology. A Personal View on Learning to Read the Book of Life. Dordrecht (The Netherlands): Springer, 2011.

Chela-Flores, J., A Second Genesis: Stepping stones towards the intelligibility of nature. Singapore: World Scientific Publishers, 2009.

Chela-Flores, J., "Astrobiological reflections on faith and reason. The Issues of Agnosticsm, Relativism and Natural Selection», en "SECKBACH, J., FORDON, R. (eds.), Divine Action and Natural Selection: Science, Faith and Evolution. Singapore: World Scientific Publishers, 2008, pp, 48-63.

Chela-Flores, J., "La posibilidad de existencia de vida extraterrestre inteligente, su búsqueda científica e interés filosófico», en «Astrobiología y Filosofía (III)», Letras de Deusto (2008) Vol. 38, no 118, pp. 38-47.

Chela-Flores, J., «La evolución del comportamiento inteligente: ¿Existe evidencia de propósito en las moléculas complejas de la química?», en «Astrobiología y Filosofía (II)», Letras de Deusto (2006) Vol. 36, n 110, pp. 21-29.

Chela-Flores, J., "Fitness of the Universe for a Second Genesis: Is it Compatible with Science and Christianity?», Science and Christian Belief (2005) Vol. 12, n 2, pp 187197.

Chela-Flores, J., "Marco cultural de la astrobiología» en "Astrobiología y Filosofía», Letras de Deusto (2003) Vol. 33, nº 98, pp. 199-215.

Chela-Flores, J., "Testing the Drake Equation in the solar sistem», en Lemarchand, G, Meech, K. J. (eds.), Bioastronomy'99: A New Era in Bioastronomy. San Francisco: Astronomical Society of the Pacific. Conference Series, Vol. 213, 2000, pp. 402-410.

Снува, C. F., Hand, K. P., «Astrobiology: the study of living universe», Annual Review of Astronomy \& Astrophysics (2005) Vol. 43, pp. 31-74.

Cirkovic, M., "Counterfactuals and unphysical ceteris paribus: An explanatory fallacy», Philosophy and Society (2013) Vol. XXIV, no 4, pp. 143-160.

Cirkovic, M., The Astrobiological Landscape. Philosophical Foundations of the Study of Cosmic Life. New York: Cambridge University Press, 2012.

Cirkovic, M., Vukotic, B., Dragievic, I., «Galactic Punctuated Equilibrium: How to Undermine Carter's Anthropic Argument in Astrobiology», Astrobiology (junio 2009) Vol. 9, n' 5 , pp 491-501.

Cirkovic, M., "Catastrophism, Fine-Tuning and changing views of extraterrestrial life and intelligence», Publ. Astronomical Observatory of Belgrade (2008) no 85, pp. 45-50.

Cirkovic, M., "Too early? on the apparent conflict of astrobiology and cosmology», en Biology and Philosophy. Springer (2006), Vol 21, pp. 369-379.

Cirkovic, M., «The Temporal Aspect of the Drake Ecuation», Astrobiology (2004) vol. 4, $\mathrm{n}^{\circ} 2$, pp. 225-231.

Cirkovic, M., «On the First Anthropic Argument in Astrobiology», Earth, Moon and Planets (2003) no 91, pp. 225-231.

Conway Morris, S., Life's Solution: Inevitable Humans in a Lonely Universe. Cambridge: Cambridge University Press, 2003.

Crowe, M., The Extraterrestrial Life Debate, Antiquity to 1915: A Source Book. Notre Dame: University of Notre Dame Press, 2008.

CRowe, M., «A History of the Extraterrestrial Life Debate», Zygon (1997), vol. 32, n 2, pp. 147-162. 
CRowe, M., The Extraterrestrial Life Debate, 1750-1900: The idea of plurality of worlds from Kant to Lowell. Cambridge and N.Y.: Cambridge University Press, 1986.

Czyzewska, U. K., «Difficulties of the Re-Emergent Science - the Case of Astrobiology», ELBA Bioflux (2011), vol. 3, n $\mathrm{n}^{\circ}$, pp. 27-37.

CzyzewsKa, U. K., "Methodological Aspects of Astrobiology as a Multidisciplinary Science», Astrobiology Science Conference (2010): Evolution and Life surviving Catastrophes and Extremes on Earth and Beyond (2010).

Davies, P., Un silencio inquietante. La nueva búsqueda de vida extraterrestre. Barcelona: Crítica, 2011.

Davies, P., Benner, S., Cleland, C., Lineweaver, C., McKay, C., Wolfe-Simon, F., «Signatures of a Shadow Biosphere», Astrobiology (abril, 2009) Vol. 9, n 2, pp. 241-249.

Davies, P., El quinto milagro, la búsqueda del origen y significado de la vida. Barcelona: Crítica, 2006.

Davies, P., Are We Alone? Philosophical Implications of the Discovery of Extraterrestrial Life. New York: Basic Books, 1995.

De Duve, C., Life Evolving Molecules Mind and Meaning. New York: OUP, 2002.

De Duve, C., Vital Dust: Life as a Cosmic Imperative. New York: Basic Books, 1995.

Des Marais, D. J., Nuth, J. A., et al., «The NASA Astrobiology Roadmap», Astrobiology (2008) Vol. 8, no 4, pp. 715-730.

Dick, S. J., "Critical issues in the history, philosophy, and sociology of astrobiology", Astrobiology (octubre 2012) Vol. 12, n 10, pp. 906-927.

Dick, S. J., «The Postbiological Universe», Acta Astronautica (abril-mayo 2008), Vol. 62, $n^{\circ} 8-9$, pp. 499-504.

Dick, S. J., "Cosmotheology revisited: theological implications of extraterrestrial life», Cons-Ciências: actas do Fórum Internacional Ciência, Religião e Consciência. Porto, Edições Universidade Fernando Pessoa, CTEC, 2005, pp. 287-301.

Dick, S. J., StRick, J., The living universe: NASA and the development of astrobiology. New Brunswick (New Jersey).: Rutgers University Press, 2004.

Dick, S. J. (ed.) Many Worlds: The New Universe, Extraterrestrial Life, and its Theological Implications. Philadelphia: Templeton Press, 2000.

Dick, S. J., Life on other worlds: the $20^{\text {th }}$-century extraterrestrial life debate. New York: Cambridge University Press, 1998.

Dick, S. J., The biological universe: the twenty-century extraterrestrial life debate and the limits of science. New York: Cambridge University Press, 1996.

Dick, S. J., Plurality of worlds: the origin of the extraterrestrial life debate from Democritus to Kant. New York: Cambridge University Press 1982.

Dole, S. H., Planetas habitables. Barcelona: Labor, 1973.

DraKe, F., «Nuevos paradigmas para SETI», en «Astrobiología y Filosofía (IV)», Letras de Deusto (2012) Vol. 42, n 134, pp. 23-28.

Drake, F., Sobel, D., Is Anyone Out There? The Scientific Search for Extraterrestrial Intelligence. New York: Delacorte, 1992.

Dunér, D., et al., The History and Philosophy of Astrobiology: Perspectives on Extraterrestrial Life and Human Mind. Newcastle (UK): Cambridge Scholars Publishing, 2013.

Duquaire, H., Si los astros estuviesen habitados... Valencia: Fondo de Cultura, 1964.

Ekers, R. D., Cullers, D. K., Billingham, J., SETI 2020: A Roadmap for the Search for Extraterrestrial Intelligence. Mountain View (California): SETI Press, 2002.

GARDNER, J., El Universo inteligente. Barcelona: Robinbook, 2008.

Gardner, J., Biocosm-The New Scientific Theory of Evolution: Inteligent Life is the Architect of the Universe. Makawao (Hawaii): Inner Ocean Publishing, 2003. 
Gato-Rivera, B., «A solution to the Fermi Paradox: The Solar System, part of a Galactic Hipercivilization?», Popular Physics (07/12/2005), arXiv:physics/0512062v3 [physics. pop-ph]

Gato-Rivera, B., «Brane Worlds, The Subanthropic Principle and the Undetectability Conjecture», Popular Physics (19/08/2003), arXiv:physics/0308078v4 [physics.pop$\mathrm{ph}]$.

Gilmour, I., Stephton, M., An Introduction to Astrobiology. Cambridge: Cambridge University Press, 2004.

Giménez Cañete, A., Gómez-Elvira, J., Martín Mayorga, D. (coords.), Astrobiología. Sobre el origen y evolución de la Vida en el Universo. Madrid: CSIC-Los libros de la Catarata, 2011.

Goldsmith, D., The Quest for Extraterrestrial Life: A Book of Readings. Mill Valley (California): University Science Books, 1980.

Goldsmith, D., Owen, T., The Search for Life in the Universe. Menlo Park (California): Benjamin Cummings, 1980.

González Fairén, A., Astrobiología. Madrid: Sirius, 2004.

Grinspoon, D., Lonely Planets: The Natural Philosophy of Alien Life. New York: HarperCollins Publishers, 2003.

Guthke, K. S., The Last Frontier: Imagining Other Worlds, from the Copernican Revolution to Modern Science Fiction. Ithaca, N.Y.: Cornell University Press, 1990.

Harrison, A. A., After Contact: The Human Response to Extraterrestrial Intelligence. New York: Plenum Trade, 1997.

Henderson, L. J., The Fitness of the Environment An Enquiry into the Biological Significance of the Properties of Matter. New York: Macmillan, 1913 (Reprinted in Gloucester, Mass: Peter Smith, 1970).

Hetesi, Zs, Regály, Zs, «Old questions reassessed by modern cosmology and astrobiology», en Forgács-Dajka, E., Petrovay, K., Erdélyi, R. (eds), Proceedings in Astronomy \& Astrophysics. Budapest: PADEU, 2004, pp. 251-258.

Impey, C., Spitz, A., Stoeger, W. (eds), Encountering Life in the Universe: Ethical Foundations and Social implications of Astrobiology. Tucson (USA): The University of Arizona Press, 2013.

Jakosky, B., «Philosophical Aspects of Astrobiology», en Lemarchand, G, Meech, K.J. (eds.), Bioastronomy'99: A New Era in Bioastronomy. San Francisco: Astronomical Society of the Pacific. Conference Series, Vol. 213, 2000, pp. 661-666.

Jакоsкy, B., La búsqueda de vida en otros planetas. Madrid: Cambridge University Press, 1999.

Kolb, Vera, "Role of the observer in the scientific process in astrobiology and in defining life», en Hoover, R., Levin, G., Rozanov, A., Davies, P. (eds), Instruments, Methods and Missions for Astrobiology XIII, Proc. SPIE 7819, 2010.

KolB, V., "On the applicability of dialetheism and philosophy of identity to the definition of life» International Journal of Astrobiology (abril 2010) Vol. 9, n 2, pp.131-136.

Kolb, V., "On the applicability of the identity problem to astrobiology», en Hoover, R., Levin, G., Rozanov, A., Retherford, K. (eds), Instruments and Methods for Astrobiology and Planetary Missions XII, Proc. SPIE 74410, 2009.

Kolb, V., "On the applicability of the Aristotelian principles to the definition of life», International Journal of Astrobiology (enero 2007) Vol. 6, $\mathrm{n}^{\circ}$ 1, pp. 51-57.

Kolb, V., "On the applicability of the Darwinian principles to chemical evolution that led to life», International Journal of Astrobiology (enero 2004) Vol. 3, n 1, pp. 45-53. 
Landfester, U., Remuss, N. L., Chrogl, K. W., Worms, J. C. (eds). Humans in Outer Space - Interdisciplinary Perspectives. Wien: Springer, 2011.

Lederberg, J. (1963) «Life Beyond the Earth», Stanford Today (1963) Stanford University, California, $\mathrm{n}^{\circ} 7$, pp. 1-8.

Lederberg, J., «Exobiology: Approachess to Life Beyond the Earth», Science (1960) Vol. 132, no 3424, pp. 393-400.

Lafleur, L.J., "Astrobiology», Astronomical Society of the Pacific Leaflet (Jan. 1941), $\mathrm{n}^{\circ}$ 143, pp. 333-340.

Lemarchand, G. A, Tancredi, G. (eds.), Astrobiología: del Big Bang a las Civilizaciones. Montevideo: UNESCO, 2010.

Lemarchand, G. A., El LLamado de las Estrellas: Búsqueda de Inteligencia Extraterrestre. Buenos Aires: Lugar Editorial, 1992.

Lemonick, M., Otros mundos: la búsqueda de vida en el Universo. Barcelona: Paidós, 1999.

Lewis, J. R., The Gods Have Landed: New Religions from Other Worlds. Albany (N.Y.): State University of New Cork Press, 1995.

LozADA-ChÁvez, I., "La influencia de la teoría evolutiva biológica en la búsqueda de vida en el Universo», en «Astrobiología y Filosofía (IV)», Letras de Deusto (2012) Vol. 42, $\mathrm{n}^{\circ} 134$, pp. 49-75.

Lupisella, M., Logsdon, J., «Do we need a Cosmocentric Ethic?», International Astronautical Federation (IAF) / International Astronautical Congress (Turin, Italy), Papper IAA-97-IAA.9.2.09, 1997.

Luque, B., Ballesteros, F., Márouez, A., González, M., Agea, A., Lara, L., Astrobiología. Un puente entre el Big Bang y la vida. Tres Cantos (Madrid): Akal, 2009.

Marshall, A., "Ethics and the Extraterrestrial Environment», Journal of Applied Philosophy (1993), Vol. 10, n 2 , p. 227-236.

Martínez-Frías, J., Hochberg, D., "Classifying science and technology: two problems with the UNESCO system», Interdisciplinary Science Reviews (2007), Vol. 32, no 4 , pp. 315-319.

Mayz Vallenilla, E., «Astrofísica y Meta-Técnica», en «Astrobiología y Filosofía», Letras de Deusto (2003) Vol. 33, no 98, pp. 217-224.

McConnell, B. S., Beyond Contact: A Guide to SETI and Communicating with Alien Civilizations. Sebastopol (California): O’Reilly Media, 2001.

Miret Magdalena, E., «Ciencia astrobiológica y pensamiento cristiano», en "Astrobiología y Filosofía (III)», Letras de Deusto (2008) Vol. 38, n 118, pp. 58-67.

Monod, J., El azar y la necesidad. Ensayo sobre la filosofía natural de la biología moderna. Barcelona: Barral Editores, 1971.

NASA Technical Memorandum, Workshop on the Societal Implications of Astrobiology. Final Report, 15 December, 2000.

Pérez-Hoyos, S., "Tendencias en la habitabilidad del Universo», en "Astrobiología y Filosofía (IV)», Letras de Deusto (2012) Vol. 42, nº 134, pp. 39-48.

Pérez Laborda DE, A., "La cuestión de la vida extraterrestre a la luz de los posibles y la imposible-posibilidad», en "Astrobiología y Filosofía (III)», Letras de Deusto (2008) Vol. 38, no 118 , pp. 48-57.

Persson, E., «The Moral Status of Extraterrestrial Life», Astrobiology (octubre 2012) Vol. 12, no 10, pp. 976-984.

Philip, G. K. and Freeland, S. J., "Did Evolution Select a Nonrandom "Alphabet" of Amino Acids?», Astrobiology (abril 2011) Vol 11, n 3, pp. 235-240. 
Race, M. S, Denning, K, Bertka, C., Dick, S. J., Harrison, A., Impey, C., Mancinelli, R., et al., "Astrobiology and Society: Building an Interdisciplinary Research Community", Astrobiology (October 2012) Vol. 12, n 10, pp. 958-965.

RACE, M. S., "Communicating about the discovery of extraterrestrial life: Different searches, different issues», Acta Astronautica (2008) Vol. 62, pp. 71-78.

Randolf, R., "Reconsidering the Ethical and Theological Implications od Extraterrestrial Life», Center for Theology and Natural Sciences Bulletin (1997), Vol 17, n 3, pp. 1-8.

Regis, E. Jr. (ed.), Extraterrestrials: Science and Alien Intelligence. Cambridge: Cambridge University Press, 1985.

Rescher, N., "Problems of Alien Cognition», (cap. 3) de Unknowability: An Inquiry into the Limits of Knowledge. Lanham: Lexington Books, 2009.

RodRíguez-MANFREdi, J. A., "La innovación tecnológica en la astrobiología como motor de cambio en la mentalidad actual», en "Astrobiología y Filosofía (III)», Letras de Deusto (2008) Vol. 38, no 118, pp. 68-75.

Ruíz de Gopegui, L., Mensajeros cósmicos. Ciencia y enigma de los extraterrestres. Madrid: McGraw-Hill, 1994.

Rummel, J. D., Race, Ms., Horneck, G. (eds), COSPAR Workshop on Ethical Considerations for Planetary Protection in Space Exploration. Paris: COSPAR, 2012.

Rummel, J. D., «Planetary Protection Policy (U.S.A.)», Adv. Espace Res. COSPAR (1992), Vol. 20, n 4, pp. 129-131.

Russell, D. A., «Exponential evolution: Implications for intelligent extraterrestrial life», Advanced Space Research (1983) Vol. 3, nº 9, pp. 95-103.

SAGAN, C., Comunicación con inteligencias extraterrestres. Barcelona: Planeta, 1980.

Sagan, C., The Cosmic Connection. An Extraterrestrial Perspective. New York: Anchor Press, 1973.

Schatzman, E., Los niños de Urania. En busca de las civilizaciones extraterrestres. Barcelona: Salvat, 1994.

Schatzman, J., "Philosophical issues in the search for extraterrestrial life and intelligence», International Journal of Astrobiology (2013) Vol. 12, nº 3, pp. 259-262.

SCHNEIDER, J., «The question “Are we alone?” in different cultures», en Proceedings of the International Astronomical Union (2009) Vol. 5, pp. 213-217.

ShklovskiI, I. S., Sagan, C., Intelligent Life in the Universe. San Francisco: Holden Day, 1966.

Shneour, E. A., Ottesen, E. A., Extraterrestrial Life: An Anthology and Bibliography. Washington: National Academy of Sciences/National Research Council, 1966.

Shostak, G. S. (ed.), Progress in the Search for Extraterrestrial Life. San Francisco: Astronomical Society of the Pacific, 1995.

Simpson, G. G., «The non-prevalence of humanoids» Science (1964), Vol. 143, pp. 769775.

Spencer Jones, H., Life on Other Worlds. New York: Macmillan, 1940

Steeves, H. P., «A Quantum Magical Realism Writ Small: Astrobiology, Philosophy, and the Origin of Life», en Campbell, S., Bruno, P. W. (eds.), The Science, Politics and Ontology of Life-Philosophy. Bloomsbury Academic, 2013.

Stenger, V.J., The Fallacy of Fine-Tuning: Why the Universe is not Designed for Us. New York: Prometheus Books, 2011.

Sullivan, W. T, Baross, J. A. (eds), Planets and Life: the emerging science of astrobiology. New York: Cambridge University Press, 2007.

Sullivan, W., We are Not Alone. New York: McGraw-Hill, 1964. 
Terzian, Y., Bilson, E. (eds), El Universo de Carl Sagan. Madrid: Cambridge University Press, 1999.

Tikнov, G., Astrobiology. Moscow (Russia): Molodaya Gvardia Publishing House, 1953.

Tough, A. (ed.), When SETI Succeeds: The Impact of High-Information Contact. Washington, USA: Foundation for the Future, 2000.

Urmeneta, J., Navarrete, A., ¿Hay álguien ahí? Origen y evolución de la vida en el planeta Tierra y la búsqueda de señales de vida en el espacio. Barcelona: Océano, 2002.

Vакосн, D. A. (ed.), Archaeology, Anthropology, and Interstellar Communication. Washington D.C: NASA, 2014.

VАKосн, D. A. (ed.), Extraterrestrial Altruism. Evolution and Ethics in the Cosmos. Heidelberg: Springer, 2014.

Vакосн, D. A. (ed.), Astrobiology, History, and Society: Life Beyond Earth and the Impact of Discovery. Heidelberg: Springer, 2013.

Varela, F. J., Thompson, E, Rosch, E., The Embodied Mind: Cognitive Science and Human Experience. Cambridge: MIT Press, 1991.

Vázouez Abeledo, M., Guerrero de Escalante, E. M., La búsqueda de vida extraterrestre. Madrid: McGraw-Hill, 1999.

WeBb, S., If the Universe is Teeming with Aliens... Where is Everybody? Fifty Solutions to Fermi's Paradox and the Problem of Extraterrestrial Life. New York: Copernicus, 2002.

YNDURÁrn, F. J., ¿Quién anda ahí? Civilizaciones extraterrestres y el futuro de la humanidad. Madrid: Debate, 1997.

Universidad de Deusto, Bilbao

Roberto AretXaga-Burgos

roberto.aretxaga@deusto.es

[Artículo aprobado para publicación en diciembre de 2014] 